\title{
Proceeding
}

Supplementary Issue: Summer Conferences of Sports Science. Costa Blanca Sports Science Events, 25-26 September 2020. Alicante, Spain.

\section{A systematic review on the muscular activation on the lower limbs with five different variations of the squat exercise}

\author{
JAVIER GENE-MORALES ${ }^{1,2}$, JORGE FLANDEZ³, ALVARO JUESAS², PEDRO GARGALLO², IVÁN \\ MIÑANA², JUAN C. COLADO² \\ ${ }^{1}$ Research Institute on Traffic and Road Safety (INTRAS), University of Valencia, Valencia, Spain \\ ${ }^{2}$ Research Unit in Sport and Health, University of Valencia, Valencia, Spain \\ 3 Institute of Education Sciences, Austral University of Chile, Ciudad de Valdivia, Chile
}

\begin{abstract}
The squat is one of the most commonly used resistance exercises for performance and health due to its biomechanical and neuromuscular similarities to a wide range of athletic and everyday activities. There is a large number of squat variations (based on the descent depth, width of the stance, bar placement) with significant biomechanical and neuromuscular differences between them. The aim of this study was to systematically review the scientific literature to gather data on the muscular activation of the lower limb during different variants of the squat exercise. High-bar squat (full range of motion, to parallel and partial range of motion), low-bar squat, front squat, overhead squat and guided squat on Smith machine were included in the analysis. 30 articles met the inclusion criteria and were reviewed. Quality of the included studies was analysed with the PEDro scale. Main findings were that in the squat exercise activation of the knee-extensors is predominant. However, different activation patterns were observed with different distances between the feet, different depths, hips rotation or flexion, intensities. For instance, low-bar squat involves a greater hip hinge and thus, provokes major activation on the hip-extensors than other squat variations. It is worth highlighting that similar activation patterns were observed between the front squat and the high-bar squat. The variation with least activation was the guided squat. The evidence presented in this study may help the strength and conditioning professionals and practitioners with the exercise selection depending on the muscular targets and the individual characteristics of the athlete.
\end{abstract}

Keywords: Electromyographic activity; Resistance exercise; Quadriceps; Gluteus; Hamstrings; Calves.

Cite this article as:

Gene-Morales, J., Flandez, J., Juesas, A., Gargallo, P., Miñana, I., \& Colado, J.C. (2020). A systematic review on the muscular activation on the lower limbs with five different variations of the squat exercise. Journal of Human Sport and Exercise, 15(4proc), S1277-S1299. doi:https://doi.org/10.14198/ihse.2020.15.Proc4.28

Corresponding author. Department of Physical Education and Sports, University of Valencia. C/ Gascó Oliag 3, 46010, Valencia, Spain. https://orcid.org/0000-0002-3255-3940

E-mail: juan.colado@uv.es

Abstract submitted to: Spring Conferences of Sports Science. Costa Blanca Sports Science Events, 19-20 June 2020. Alicante, Spain.

JOURNAL OF HUMAN SPORT \& EXERCISE ISSN 1988-5202

(c) Faculty of Education. University of Alicante

doi:10.14198/jhse.2020.15.Proc4.28 


\section{INTRODUCTION}

The squat is one of the most commonly used resistance exercises for performance and health due to its biomechanical and neuromuscular similarities to a wide range of athletic and everyday activities (Andersen et al., 2016; Clark et al., 2012; Kompf \& Arandjelović, 2017; Schoenfeld, 2010). All variants of the squat involve synergistic hip, knee, and ankle flexion in the descent, followed by knee and hip extension in the ascent which finishes with the individual in the starting position (Clark et al., 2012; Escamilla et al., 2001; Iversen et al., 2017; Schoenfeld, 2010; Vigotsky et al., 2019). However, there is a large number of squat variations (based on the descent depth, the width of the stance, bar placement, orientation of the knee flexion planes, and so on) with significant biomechanical and neuromuscular differences between them (Clark et al., 2012; Kompf \& Arandjelović, 2017; Schoenfeld, 2010; Van den Tillaar et al., 2014). One of the aims of the expert research to the date has been to enlighten the strength and conditioning professionals and athletes with the differences between these variations in terms of muscular activity (Bourne et al., 2017). Understanding the muscular activity of each exercise is a key point in the prescription and programming of resistance exercises depending on the individual characteristics (Bolgla \& Uhl, 2005; Borreani et al., 2014; Neto et al., 2020).

Muscular activity is often measured with surface electromyography, a method that registers the intensity and duration of electric signals produced in the muscles (Chowdhury et al., 2013). Electrodes are placed on specific superficial points that cover the muscle to analyse. The electromyograph gives raw data in absolute electric signal intensity in millivolts $(\mathrm{mV})$ or microvolts $(\mu \mathrm{V})$. Typical methods to standardize the results are a) as a relative percentage of a maximum voluntary isometric contraction (IMVC); b) as a relative percentage of the maximum historical contraction (MVC); c) as the square root of the average power of the EMG signal for a given period of time (root mean square; RMS) (Sinclair et al., 2015). Data in the scientific literature are uneven and thus, comparisons between studies are sometimes difficult.

The main objective of this research was to systematically review the expert literature to gather data on the muscular activation of the lower limb during different variants of the squat exercise. We aimed to identify the main characteristics of each variant, the predominant muscle groups involved, and to determine the variant with higher activation levels, through the analyses of the included studies.

\section{METHODS}

For this systematic review, the protocols of the PRISMA declaration (Hutton et al., 2015; Urrútia \& Bonfill, 2010) were followed.

\section{Search strategy}

Four databases (Web of Science, PubMed, Scopus, and SportDiscus) and ProQuest (i.e. an electronic tool containing doctoral thesis) were consulted to collect information about muscular activation. Also, the Strength and Conditioning Journal was consulted. No temporal restrictions were used in the search. The following terms were used: "squat" OR "squat exercise" OR "high bar squaf" OR "low bar squat" OR "overhead squaf" OR "front squat"] AND ["EMG" OR "electromyography" OR "electromyographic activity" OR "muscle activation" OR "muscle activity"]. A third line was added with the following terms to include technical variations: ["stance width" OR "hip rotation" OR "Smith machine" OR "deep" OR "depth" OR "parallel" OR "partial" OR "quarter"]. Furthermore, the operator "NOT" was used in combination with the terms "balance", "instability", "unstable", "bands", "chains", "injury", "injured", "unload", and "therapeutic" to refine the results and exclude articles that did not follow the inclusion criteria. 


\section{Eligibility criteria}

Studies that examined muscle activation on the lower limb in squats written in Spanish or English were included in the analyses. Inclusion criteria were a) including healthy subjects with no recent history of injury; b) using stable surfaces to perform the squats; c) using a barbell with load. On the other hand, exclusion criteria were a) using variable resistance (i.e. elastic bands, chains) to load the exercise; b) analysing muscle activity of the upper limb or trunk; c) performing an isometric squat.

\section{Article selection and data processing}

\section{Studies}

Screening of titles and abstracts was initially carried out to identify potentially relevant studies. A standardized form was used to assess the eligibility of each article considering the inclusion-exclusion criteria. Figure 1 shows the flow diagram that summarizes the study selection process after the reading of the titles and abstracts of the initial search.

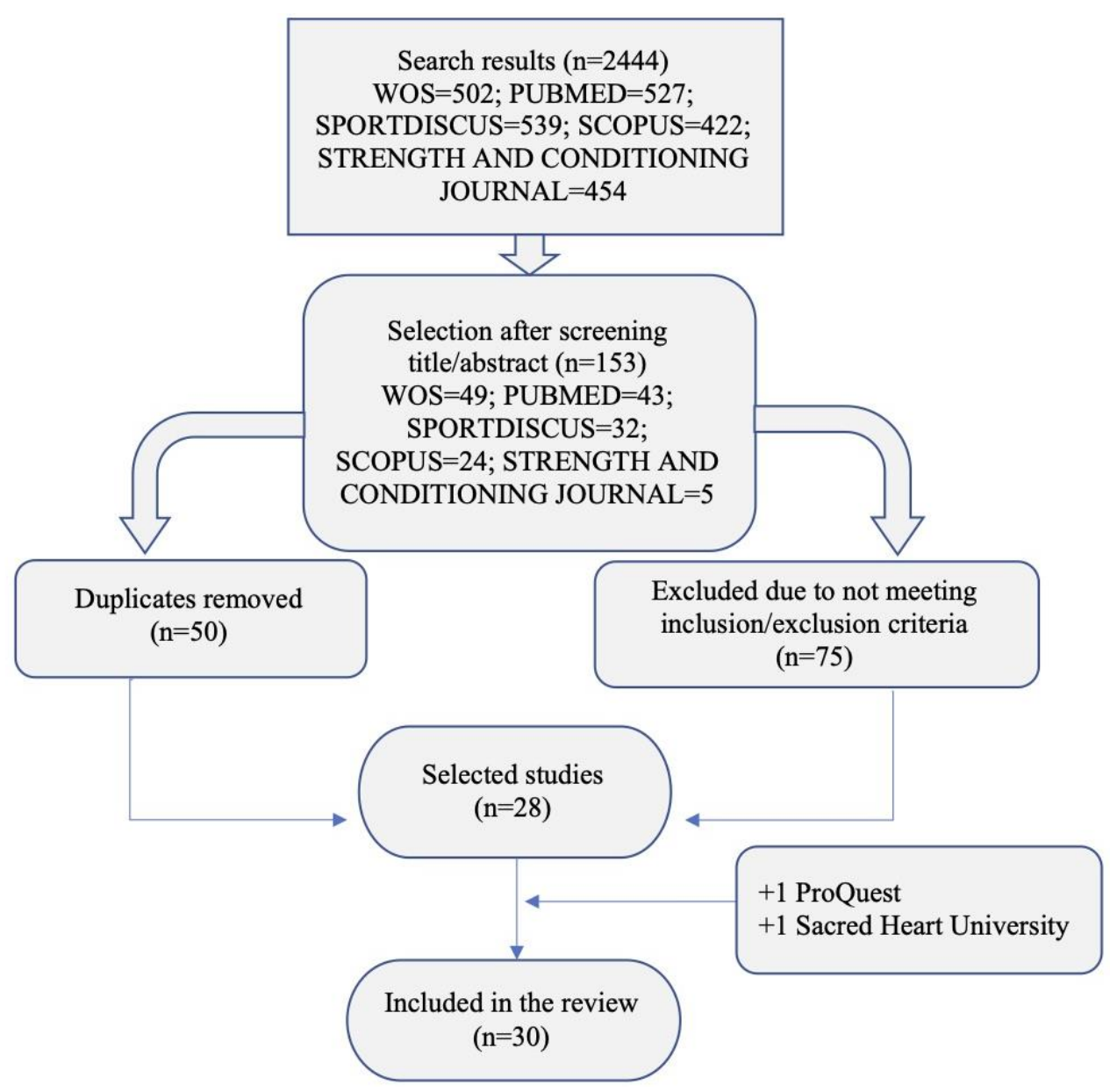

Figure 1. Flow diagram that summarizes the study selection process from the first search to the final selection.

\section{Squat variations}

After carefully reading the selected articles five squat variations were selected for the analysis by agreement between the authors. Some squat variations found in the literature and excluded from the analysis were the unilateral squat, Bulgarian squat, and wall squat. The five squat variations included were (see Figure 2): 
High-bar squat (26 studies): the bar is placed across the shoulder on the trapezius, slightly above the level of the acromion and the posterior aspect of the deltoids (Schoenfeld, 2010; Vigotsky et al., 2019; Wretenberg et al., 1996).

Front squat (5 studies): the bar is held in front of the chest at the clavicle (Bautista, 2019; Schoenfeld, 2010).

Overhead squat (2 studies): bar is held with both hands, fully extended elbows, and externally rotated shoulders (Bautista, 2019).

Guided squat on Smith machine (2 studies): the bar is guided and thus, it only can be moved up and down (different variations of the squat can be performed on the Smith machine, however for this study, we considered a high bar squat; Clark et al., 2012).

Low-bar squat (1 study): the bar is placed slightly below the level of the acromion (Schoenfeld, 2010; Wretenberg et al., 1996).

a)

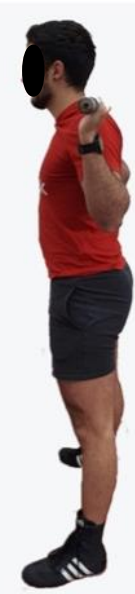

c)

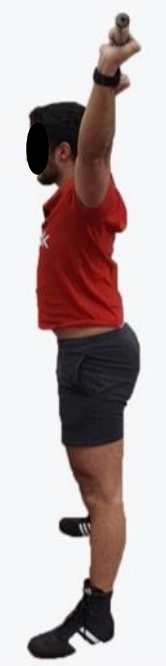

b)

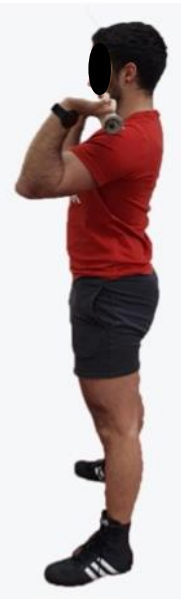

d)

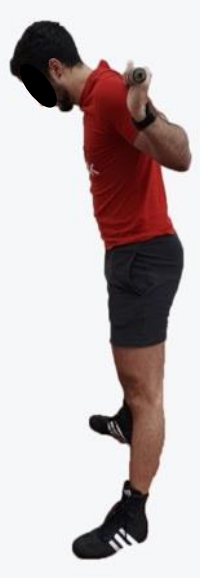

Figure 2. From left to right: a) high-bar squat, b) front-squat, c) overhead squat, d) low-bar squat. The guided squat is not pictured as for this study, the placement of the bar was the same as in the high-bar squat. 
The high bar squat was in turn divided in full range of motion (ROM; i.e. the hips are lower than the knees), to parallel (i.e. lowering until the femur is parallel to the ground, approximately $90^{\circ}$ of knee movement), and partial range of motion (i.e. half the ROM of a parallel squat, a quarter of a full ROM squat, approximately $45^{\circ}$ of knee movement). Also, different technical variants of the squat exercise such as stance width and hip rotation planes were included to enrich the analyses.

\section{Muscles analysed}

As mentioned in the objective, this study focuses on the muscles of the lower limb. After a thorough reading of the selected articles, the authors selected the muscles to be included in the analysis. These muscles were a) gluteus maximus, b) gluteus medialis, c) hip adductors, d) vastus lateralis, e) vastus medialis, f) rectus femoris, g) biceps femoris, $h$ ) semitendinosus, i) tibialis anterior, j) gastrocnemius and $k$ ) soleus. Muscles " $a$, $b$, and c" act mainly on the hips; muscles " $d, e$, and f' are part of the quadriceps and are mainly involved in the knee-extension; muscles " $g$ and $h$ " are part of the hamstrings and their contraction mainly affect the knees; the tibialis anterior (muscle " $i$ ") is an ankle dorsiflexor; and finally, the muscles " $j$ and $k$ " are part of the calves and their action provoke an ankle extension. Previous expert literature (Netter, 1999) can be consulted for further information on the included muscles and anatomy.

\section{Electromyographic values}

EMG values are unequally reported among the expert literature, not only on the units used (millivolts, microvolts, percentage of isometric maximum voluntary contraction, percentage of maximum voluntary contraction, percentage of root mean square values) but also on the measured phase (concentric and eccentric, mean of the set, mean of a repetition). In this review, the authors standardized the values when possible to facilitate the comprehension and the comparison between studies. For instance, values in millivolts were transformed into microvolts. Also, results of concentric and eccentric phases were averaged to obtain a single value.

\section{Quality assessment}

The quality of the included studies was analysed using the PEDro scale (Maher et al., 2003). The scale was modified to fit the design of the included studies (see Table 3). Points 2 and 3 were unified into one point that assessed the randomization of the exercise conditions performed. Point 4 had to be excluded due to not including studies with control and experimental groups. Finally, points 5, 6, and 7 were also excluded due to the impossibility of blinding subjects or researchers. The resultant scale to evaluate the quality of the articles was composed of 6 items.

\section{RESULTS}

30 articles met the inclusion criteria and were reviewed (Figure 1). As abovementioned (see "data processing and analysis-squat variations" section), the most widely studied variation of the squat exercise was the highbar squat ( 26 studies), followed by the front-squat ( 5 studies), the overhead squat, and guided squat (both analysed by 2 studies), and finally the low-bar squat (1 study). The main muscular group involved in all the variations was the quadriceps, with some differences between each squat variation, and also between different technical modifications (i.e. ROM, stance width, hip rotation, feet placement).

Due to the considerable amount of variations and exercise conditions, the results section will be divided into six subsections: one for each squat variation, and one including the technical modifications of the exercise (i.e. stance width, hip rotation). Furthermore, Table 1 includes the main characteristics of the included studies (i.e. sample characteristics, exercise condition, measured muscles, and main results), and Table 2 presents 
reported EMG values. EMG values are presented in different units (i.e. absolute values, IMVC, MVC, RMS), and thus caution should be applied when comparing values between studies.

\section{High-bar squat}

A major part of the studies found the main activity on the vastus lateralis, vastus medialis, and rectus femoris, in this order (Aspe \& Swinton, 2014; Contreras et al., 2015, 2016; da Silva et al., 2017; Delgado et al., 2019; Ebben et al., 2009; Eliassen et al., 2018; Gorsuch et al., 2013; Hammond et al., 2016; Iversen et al., 2017; Korak et al., 2018; Robbins, 2011; Schwanbeck et al., 2009; Wu et al., 2019; Yavuz et al., 2015). Only one study found major activation on the biceps femoris than on each of these three aforementioned muscles (Andersen et al., 2014). Regarding the activation on the gluteus and hamstrings, while some authors observed a greater activation on the gluteus maximus (Caterisano et al., 2002; Fauth et al., 2010; McCurdy et al., 2018), others reported a higher activity on the hamstrings (Andersen et al., 2014; Delgado et al., 2019; Gullett et al., 2009). Only three authors reported activation levels on the muscles of the calves when performing a high-bar squat (Aspe \& Swinton, 2014; da Silva et al., 2017; Schwanbeck et al., 2009).

\section{Front squat}

Muscle activity in this squat variation followed similar patterns to other squat variations (i.e. major activation on the vastus lateralis and medialis). Korak et al. (2018) reported similar activation levels on the rectus femoris and gluteus maximus. Opposite to the aforementioned authors, Gullet et al. (2009) found higher activation levels on the hamstrings (semitendinosus: 140\% of the IMVC) than on the quadriceps (vastus lateralis: $60 \%$ of the IMVC; vastus medialis: $81 \%$ of the IMVC; rectus femoris: $59 \%$ of the IMVC).

\section{Overhead squat}

Two studies analysed this variation of the squat exercise (Aspe \& Swinton, 2014; Bautista, 2019). As happened with the rest of the variations, major activation levels were found on the vastus lateralis in both studies. As secondary muscles, Aspe \& Swinton (2014) reported higher activation levels on the gluteus $(61 \%$ of the MVC) than on the biceps femoris (54\% of the MVC). Bautista (2019) found lower activation levels on the biceps femoris ( $31 \%$ of the IMVC) but did not analyse the gluteus maximus.

\section{Guided squat on Smith machine}

In one study, activation patterns were the same as those reported in the high-bar squat (Schwanbeck et al., 2009). In the study of Blanpied et al. (1999) major activation levels were observed on the gluteus maximus than on the quadriceps or the hamstring. The effects of a technical modification (i.e. feet placed in line with the body or ahead) when performing a guided squat are presented in the following section.

\section{Low-bar squat}

The study of McCaw and Melrose (1999) was the only one that analysed the activation levels in a low-bar squat and met the eligibility criteria. The main activity was observed on the vastus lateralis and medialis, followed by the rectus femoris. Lower activity on the hip adductors and gluteus in comparison to the quadriceps was observed, the activity of these last two muscles being similar. The lowest activation levels were detected on the biceps femoris. Further analyses of this squat variation were based on technical modifications as shown further below (see "technical modifications" section).

\section{Technical modifications}

Range of motion

The comparison between three different depths (i.e. partial, parallel, and full squats) yielded similar activation patterns, with controversial results observed on the gluteus activation. While Caterisano et al. (2002) 
Table 1. Characteristics of the included studies $(N=30)$.

\begin{tabular}{|c|c|c|c|c|c|}
\hline (Author, year) & $\begin{array}{l}\text { Sample (N, sex, age } \\
\text { characteristics) }\end{array}$ & Exercise/s & Load & Measured muscles & Main results \\
\hline (Bautista, 2019) & $\begin{array}{l}7 \text { trained males } \\
\text { Age: } 28.0 \pm 3.6 \text { years } \\
\text { Height: } 175.0 \pm 5.3 \mathrm{~cm} \\
\text { Weight: } 92.0 \pm 26.1 \mathrm{~kg}\end{array}$ & $\begin{array}{l}\text { - Front squat } \\
\text { - Overhead } \\
\text { squat }\end{array}$ & $\begin{array}{l}3 \mathrm{R} \text { at } 65 \%, \\
80 \% \text { and } \\
95 \% \text { of } 3 \mathrm{RM}\end{array}$ & $\begin{array}{l}\text { - Vastus lateralis } \\
\text { - Biceps femoris }\end{array}$ & $\begin{array}{l}\text { Major activity on the vastus lateralis in the concentric } \\
\text { phase of the front squat, being this major activity on the } \\
\text { vastus lateralis in the eccentric phase of the overhead } \\
\text { squat. }\end{array}$ \\
\hline $\begin{array}{l}\text { (Delgado et al., } \\
\text { 2019) }\end{array}$ & $\begin{array}{l}8 \text { trained males } \\
\text { Age: } 25.0 \pm 3.3 \text { years } \\
\text { Height: } 177.7 \pm 6.6 \mathrm{~cm} \\
\text { Weight: } 84.0 \pm 6.5 \mathrm{~kg} \\
\text { Minimum experience: } 1 \\
\text { year }\end{array}$ & $\begin{array}{l}\text { - Full ROM high- } \\
\text { bar squat }\end{array}$ & 1RM & $\begin{array}{l}\text { - Gluteus maximus } \\
\text {-Vastus lateralis } \\
\text { - Biceps femoris }\end{array}$ & Major activity on the vastus lateralis. \\
\hline (Wu et al., 2019) & $\begin{array}{l}19 \text { trained males } \\
\text { Age } 22.1 \pm 1.1 \text { years } \\
\text { Height: } 174.4 \pm 5.2 \mathrm{~cm} \\
\text { Weight: } 76 \pm 13.3 \mathrm{~kg}\end{array}$ & $\begin{array}{l}\text { - High-bar squat } \\
\text { to parallel }\end{array}$ & $\begin{array}{l}\text { 10R with } \\
20 \mathrm{~kg}\end{array}$ & $\begin{array}{l}\text { - Vastus medialis } \\
\text { - Vastus lateralis } \\
\text { - Rectus femoris } \\
\text { - Biceps femoris } \\
\text { - Semitendinosus } \\
\text { - Tibialis anterior } \\
\text { - Gastrocnemius }\end{array}$ & Major activity on the vastus lateralis and medialis. \\
\hline $\begin{array}{l}\text { (Korak et al., } \\
\text { 2018) }\end{array}$ & $\begin{array}{l}13 \text { females } \\
\text { Age: } 22.8 \pm 1.0 \text { years } \\
\text { Height: } 166.4 \pm 4.2 \mathrm{~cm} \\
\text { Weight: } 73.4 \pm 14 \mathrm{~kg} \\
\text { Minimum experience: } 1 \\
\text { year }\end{array}$ & $\begin{array}{l}\text { - High-bar squat } \\
\text { to parallel } \\
\text { - Front squat to } \\
\text { parallel }\end{array}$ & $\begin{array}{l}3 \mathrm{R} \text { at } \\
75 \% 1 \mathrm{RM}\end{array}$ & $\begin{array}{l}\text { - Gluteus maximus } \\
\text { - Vastus medialis } \\
\text { - Rectus femoris } \\
\text { - Biceps femoris }\end{array}$ & $\begin{array}{l}\text { - Back squat: Major activation on the rectus femoris, vastus } \\
\text { lateralis, and vastus medialis. } \\
\text { - Front squat: Major activation on the vastus lateralis, } \\
\text { vastus medialis, rectus femoris, and gluteus maximus. }\end{array}$ \\
\hline $\begin{array}{l}\text { (Eliassen et al., } \\
\text { 2018) }\end{array}$ & $\begin{array}{l}14 \text { trained males } \\
\text { Age: } 23.0 \pm 4.0 \text { years } \\
\text { Height: } 181.0 \pm 6.0 \mathrm{~cm} \\
\text { Weight: } 80.5 \pm 8.5 \mathrm{~kg}\end{array}$ & $\begin{array}{l}\text { - High-bar partial } \\
\text { squat }\end{array}$ & 4RM & $\begin{array}{l}\text { - Gluteus maximus } \\
\text { - Gluteus medialis } \\
\text { - Vastus lateralis } \\
\text { - Vastus medialis } \\
\text { - Rectus femoris } \\
\text { - Biceps femoris } \\
\text { - Semitendinosus } \\
\text { - Gastrocnemius }\end{array}$ & $\begin{array}{l}\text { Major activity on the rectus femoris, vastus lateralis, and } \\
\text { vastus medialis. }\end{array}$ \\
\hline $\begin{array}{l}\text { (McCurdy et al., } \\
\text { 2018) }\end{array}$ & $\begin{array}{l}18 \text { females } \\
\text { Age: } 20.9 \pm 1.1 \text { years } \\
\text { Height: } 165.0 \pm 5.5 \mathrm{~cm} \\
\text { Weight: } 61.8 \pm 6.4 \mathrm{~kg} \\
\text { Minimum experience: } 1 \text { - } \\
\text { 5years }\end{array}$ & $\begin{array}{l}\text { - High-bar squat } \\
\text { to parallel }\end{array}$ & $\begin{array}{l}\text { 3R at 8RM } \\
\text { load }\end{array}$ & $\begin{array}{l}\text { - Gluteus maximus } \\
\text { - Hamstrings }\end{array}$ & Major activation on the gluteus maximus. \\
\hline
\end{tabular}




\begin{tabular}{|c|c|c|c|c|c|}
\hline $\begin{array}{l}\text { (Iversen et al., } \\
2017)\end{array}$ & $\begin{array}{l}12 \text { males and } 12 \text { females } \\
\text { Age: } 25.0 \pm 3.0 \text { and } \\
25.0 \pm 2.0 \text { years }\end{array}$ & $\begin{array}{l}\text { - High-bar squat } \\
\text { to parallel }\end{array}$ & $\begin{array}{l}3 R \text { at } 10 R M \\
\text { load }\end{array}$ & $\begin{array}{l}\text { - Gluteus maximus } \\
\text { - Vastus medialis } \\
\text { - Vastus lateralis } \\
\text { - Rectus femoris } \\
\text { - Biceps femoris } \\
\text { - Semitendinosus }\end{array}$ & Major activation on the vastus medialis and lateralis. \\
\hline $\begin{array}{l}\text { (da Silva et al., } \\
\text { 2017) }\end{array}$ & $\begin{array}{l}15 \text { males } \\
\text { Age: } 26.0 \pm 5.0 \text { years } \\
\text { Height: } 173.0 \pm 6.0 \mathrm{~cm}\end{array}$ & $\begin{array}{l}\text { - High-bar squat } \\
\text { to parallel } \\
\text { - Full ROM high- } \\
\text { bar squat }\end{array}$ & 10RM & $\begin{array}{l}\text { - Gluteus maximus } \\
\text { - Vastus medialis } \\
\text { - Vastus lateralis } \\
\text { - Rectus femoris } \\
\text { - Biceps femoris } \\
\text { - Semitendinosus } \\
\text { - Soleus }\end{array}$ & $\begin{array}{l}\text { Major activity on the vastus lateralis and medialis, and } \\
\text { rectus femoris. Similar activation patterns in both high-bar } \\
\text { squat variants. }\end{array}$ \\
\hline $\begin{array}{l}\text { (Contreras et al., } \\
\text { 2016) }\end{array}$ & $\begin{array}{l}13 \text { females } \\
\text { Age: } 28.9 \pm 5.1 \text { years } \\
\text { Height: } 164.0 \pm 6.3 \mathrm{~cm} \\
\text { Weight: } 58.2 \pm 6.4 \mathrm{~kg}\end{array}$ & $\begin{array}{l}\text { - High-bar squat } \\
\text { to parallel } \\
\text { - Full ROM high- } \\
\text { bar squat } \\
\text { - Front squat }\end{array}$ & 10RM & $\begin{array}{l}\text { - Gluteus maximus } \\
\text { - Vastus lateralis } \\
\text { - Biceps femoris }\end{array}$ & $\begin{array}{l}\text { Major activation on the vastus lateralis in all the three } \\
\text { variations of the squat exercise. }\end{array}$ \\
\hline $\begin{array}{l}\text { (Hammond et } \\
\text { al., 2016) }\end{array}$ & $\begin{array}{l}8 \text { males } \\
\text { Age: } 21.0 \pm 1.0 \text { years } \\
\text { Height: } 176.0 \pm 5.0 \mathrm{~cm} \\
\text { Weight: } 80.0 \pm 9.0 \mathrm{~kg} \\
\text { Minimum experience } \\
5.0 \pm 1.0 \text { years }\end{array}$ & $\begin{array}{l}\text { - High-bar partial } \\
\text { squat } \\
\text { - High-bar squat } \\
\text { to parallel } \\
\text { - Full ROM high- } \\
\text { bar squat }\end{array}$ & $5 \mathrm{RM}$ & $\begin{array}{l}\text { - Gluteus maximus } \\
\text { - Vastus medialis } \\
\text { - Vastus lateralis } \\
\text { - Biceps femoris }\end{array}$ & $\begin{array}{l}\text { - Major activation on the vastus medialis and vastus } \\
\text { lateralis in all the three variants of the exercise. } \\
\text { - Minor general muscle activity in the partial squat. }\end{array}$ \\
\hline $\begin{array}{l}\text { (Contreras et al., } \\
\text { 2015) }\end{array}$ & $\begin{array}{l}13 \text { females } \\
\text { Age: } 28.9 \pm 5.1 \text { years } \\
\text { Height: } 164.0 \pm 6.3 \mathrm{~cm} \\
\text { Weight: } 58.2 \pm 6.4 \mathrm{~kg}\end{array}$ & $\begin{array}{l}\text { - High-bar squat } \\
\text { to parallel }\end{array}$ & 10RM & $\begin{array}{l}\text { - Gluteus maximus } \\
\text { - Vastus lateralis } \\
\text { - Biceps femoris }\end{array}$ & Major activation on the vastus lateralis. \\
\hline $\begin{array}{l}\text { (Yavuz et al., } \\
\text { 2015) }\end{array}$ & $\begin{array}{l}19 \text { women, } 9 \text { men, } 21.563 \\
\text { years, } 17068.4 \mathrm{~cm}, 65.7 \\
611.8 \mathrm{~kg} \\
19 \text { women, } 9 \text { men, } 21.563 \\
\text { years, } 17068.4 \mathrm{~cm}, 65.7 \\
611.8 \mathrm{~kg} \\
12 \text { males } \\
\text { Age: } 21.2 \pm 1.9 \text { years }\end{array}$ & $\begin{array}{l}\text { - High-bar squat } \\
\text { to parallel } \\
\text { - Front squat }\end{array}$ & $\begin{array}{l}1 \mathrm{R} \text { at } \\
90 \% 1 \mathrm{RM}\end{array}$ & $\begin{array}{l}\text { - Gluteus maximus } \\
\text { - Vastus medialis } \\
\text { - Vastus lateralis } \\
\text { - Rectus femoris } \\
\text { - Biceps femoris } \\
\text { - Semitendinosus }\end{array}$ & $\begin{array}{l}\text { Major activation on the vastus medialis and vastus lateralis } \\
\text { in both exercises. Secondary activation on: } \\
\text { - Back squat: Gluteus maximus and rectus femoris. } \\
\text { - Front squat: Rectus femoris. }\end{array}$ \\
\hline $\begin{array}{l}\text { (Aspe \& } \\
\text { Swinton, 2014) }\end{array}$ & $\begin{array}{l}14 \text { males } \\
\text { Age: } 26.0 \pm 7.0 \text { years } \\
\text { Height: } 182.5 \pm 13.5 \mathrm{~cm} \\
\text { Weight: } 90.5 \pm 17.5 \mathrm{~kg}\end{array}$ & $\begin{array}{l}\text { - Full ROM high- } \\
\text { bar squat } \\
\text { - Overhead } \\
\text { squat }\end{array}$ & $\begin{array}{l}3 \mathrm{R} \text { at } 60 \%, \\
75 \% \text { and } \\
90 \% \text { of } 1 \mathrm{RM}\end{array}$ & $\begin{array}{l}\text { - Gluteus maximus } \\
\text { - Vastus lateralis } \\
\text { - Biceps femoris } \\
\text { - Gastrocnemius }\end{array}$ & $\begin{array}{l}\text { Major activity on the vastus lateralis in both squat } \\
\text { variations. }\end{array}$ \\
\hline
\end{tabular}

S1284 | 2020| Proc4| VOLUME 15

C 2020 University of Alicante 


\begin{tabular}{|c|c|c|c|c|c|}
\hline $\begin{array}{l}\text { (Van den Tillaar } \\
\text { et al., 2014) }\end{array}$ & $\begin{array}{l}15 \text { trained males } \\
\text { Age: } 24.0 \pm 4.0 \text { years } \\
\text { Height: } 179.0 \pm 6.0 \mathrm{~cm} \\
\text { Weight: } 82.0 \pm 11.0 \mathrm{~kg} \\
\text { Minimum experience: } \\
6.0 \pm 3.0 \text { years }\end{array}$ & $\begin{array}{l}\text { - High-bar squat } \\
\text { to parallel }\end{array}$ & 6RM & $\begin{array}{l}\text { - Vastus medialis } \\
\text { - Vastus lateralis } \\
\text { - Rectus femoris } \\
\text { - Biceps femoris }\end{array}$ & Major activity on the vastus lateralis and vastus medialis. \\
\hline $\begin{array}{l}\text { (Andersen et al., } \\
\text { 2014) }\end{array}$ & $\begin{array}{l}15 \text { trained males } \\
\text { Age: } 24.0 \pm 4.0 \text { years } \\
\text { Height: } 179.0 \pm 6.0 \mathrm{~cm} \\
\text { Weight: } 82.0 \pm 11.0 \mathrm{~kg} \\
\text { Minimum experience: } \\
6.0 \pm 3.0 \text { years }\end{array}$ & $\begin{array}{l}\text { - High-bar squat } \\
\text { to parallel }\end{array}$ & 6RM & $\begin{array}{l}\text { - Vastus medialis } \\
\text { - Vastus lateralis } \\
\text { - Rectus femoris } \\
\text { - Biceps femoris } \\
\text { - Soleus }\end{array}$ & Major activation on the biceps femoris. \\
\hline $\begin{array}{l}\text { (Gorsuch et al., } \\
\text { 2013) }\end{array}$ & $\begin{array}{l}10 \text { males and } 10 \text { females } \\
\text { Age: } 19.2 \pm 0.4 \text { and } \\
19.9 \pm 0.4 \text { years } \\
\text { Height: } 176.8 \pm 1.5 \text { and } \\
166.7 \pm 1.5 \mathrm{~cm} \\
\text { Weight: } 66.2 \pm 2.5 \text { and } \\
55.9 \pm 1.4 \mathrm{~kg}\end{array}$ & $\begin{array}{l}\text { - High-bar squat } \\
\text { to parallel } \\
\text { - High-bar partial } \\
\text { squat }\end{array}$ & $\begin{array}{l}6 \mathrm{R} \text { at } 10 \mathrm{RM} \\
\text { load }\end{array}$ & $\begin{array}{l}\text { - Rectus femoris } \\
\text { - Biceps femoris } \\
\text { - Gastrocnemius }\end{array}$ & Major activation on rectus femoris in both exercises. \\
\hline $\begin{array}{l}\text { (Lynn \& Noffal, } \\
\text { 2012) }\end{array}$ & $\begin{array}{l}15 \text { males and } 16 \text { females } \\
\text { Age: } 23.1 \pm 2.1 \text { years } \\
\text { Height: } 170.0 \pm 11.0 \mathrm{~cm} \\
\text { Weight: } 71.0 \pm 17.3 \mathrm{~kg}\end{array}$ & $\begin{array}{l}\text { - Full ROM high- } \\
\text { bar squat }\end{array}$ & $\begin{array}{l}\text { As many } \\
\text { repetitions in } \\
\text { one minute }\end{array}$ & $\begin{array}{l}\text { - Gluteus maximus } \\
\text { - Rectus femoris } \\
\text { - Biceps femoris }\end{array}$ & Major activation on the rectus femoris and vastus. \\
\hline (Robbins, 2011) & $\begin{array}{l}10 \text { males } \\
\text { Age: } 24.0 \pm 1.2 \text { years } \\
\text { Height: } 177.0 \pm 5.0 \mathrm{~cm} \\
\text { Weight: } 82.2 \pm 10.2 \mathrm{~kg}\end{array}$ & $\begin{array}{l}\text { - High-bar squat } \\
\text { to parallel }\end{array}$ & $\begin{array}{l}3 \mathrm{R} \text { at } \\
85 \% 1 \mathrm{RM}\end{array}$ & $\begin{array}{l}\text { - Gluteus maximus } \\
\text { - Vastus medialis } \\
\text { - Biceps femoris } \\
\text { - Gastrocnemius }\end{array}$ & Major activation on the vastus medialis. \\
\hline $\begin{array}{l}\text { (Pereira et al., } \\
\text { 2010) }\end{array}$ & $\begin{array}{l}5 \text { males and } 5 \text { females } \\
\text { Age: } 21.0 \pm 1.0 \text { years } \\
\text { Height: } 171.4 \pm 9.4 \mathrm{~cm} \\
\text { Weight: } 66.5 \pm 11.4 \mathrm{~kg}\end{array}$ & $\begin{array}{l}\text { - High-bar squat } \\
\text { to parallel (three } \\
\text { hip rotations) }\end{array}$ & 10RM & $\begin{array}{l}\text { - Hip adductors } \\
\text { - Rectus femoris }\end{array}$ & $\begin{array}{l}\text { The more external rotation more activation on the hip } \\
\text { adductors, but no changes on rectus femoris. }\end{array}$ \\
\hline $\begin{array}{l}\text { (Fauth et al., } \\
2010)\end{array}$ & $\begin{array}{l}16 \text { females } \\
\text { Age: } 21.2 \pm 2.2 \text { years } \\
\text { Height: } 169.4 \pm 7.5 \mathrm{~cm} \\
\text { Weight: } 66.1 \pm 9.9 \mathrm{~kg}\end{array}$ & $\begin{array}{l}\text { - Full ROM high- } \\
\text { bar squat }\end{array}$ & $\begin{array}{l}2 \mathrm{R} \text { at } 6 \mathrm{RM} \\
\text { load }\end{array}$ & $\begin{array}{l}\text { - Gluteus maximus } \\
\text { - Gluteus medialis } \\
\text { - Vastus medialis } \\
\text { - Vastus lateralis } \\
\text { - Rectus femoris } \\
\text { - Biceps femoris } \\
\text { - Semitendinosus }\end{array}$ & $\begin{array}{l}\text { Major activation on the vastus lateralis and medialis, and } \\
\text { gluteus maximus. }\end{array}$ \\
\hline
\end{tabular}




\begin{tabular}{|c|c|c|c|c|c|}
\hline $\begin{array}{l}\text { (Ebben et al., } \\
\text { 2009) }\end{array}$ & $\begin{array}{l}11 \text { males and } 9 \text { females } \\
\text { Age: } 21.5 \pm 1.9 \text { and } \\
20.0 \pm 1.5 \text { years } \\
\text { Weight: } 78.9 \pm 9.6 \text { and } \\
66.4 \pm 7.5 \mathrm{~kg}\end{array}$ & $\begin{array}{l}\text { - Full ROM high- } \\
\text { bar squat }\end{array}$ & $\begin{array}{l}2 \mathrm{R} \text { at } 6 \mathrm{RM} \\
\text { load }\end{array}$ & $\begin{array}{l}\text { - Vastus lateralis } \\
\text { - Rectus femoris } \\
\text { - Biceps femoris }\end{array}$ & Major activation on the vastus lateralis and rectus femoris. \\
\hline $\begin{array}{l}\text { (Schwanbeck et } \\
\text { al., 2009) }\end{array}$ & $\begin{array}{l}3 \text { males and } 3 \text { females } \\
\text { Age: } 22.0 \pm 1.2 \text { years } \\
\text { Height: } 171.0 \pm 12.0 \mathrm{~cm} \\
\text { Weight: } 71.5 \pm 12.7 \mathrm{~kg} \\
\text { Minimum experience: } 2-5 \\
\text { years }\end{array}$ & $\begin{array}{l}\text { - Guided squat } \\
\text { on Smith } \\
\text { machine } \\
\text { - High-bar squat } \\
\text { to parallel }\end{array}$ & 8RM & $\begin{array}{l}\text { - Vastus medialis } \\
\text { - Vastus lateralis } \\
\text { - Biceps femoris } \\
\text { - Tibialis anterior } \\
\text { - Gastrocnemius }\end{array}$ & $\begin{array}{l}\text { Major general activation in the free-weight squat than in the } \\
\text { guided squat. } \\
\text { Major activation on the vastus lateralis and medialis in both } \\
\text { variations of the squat exercise }\end{array}$ \\
\hline $\begin{array}{l}\text { (Gullett et al., } \\
2009)\end{array}$ & $\begin{array}{l}9 \text { males and } 6 \text { females } \\
\text { Age: } 22.1 \pm 3.6 \text { years } \\
\text { Height: } 171.2 \pm 6.4 \mathrm{~cm} \\
\text { Weight: } 69.7 \pm 6.2 \mathrm{~kg}\end{array}$ & $\begin{array}{l}\text { - High-bar squat } \\
\text { to parallel } \\
\text { - Front squat }\end{array}$ & $\begin{array}{l}3 \mathrm{R} \text { at } \\
70 \% 1 \mathrm{RM} \\
\text { load }\end{array}$ & $\begin{array}{l}\text { - Vastus medialis } \\
\text { - Vastus lateralis } \\
\text { - Rectus femoris } \\
\text { - Biceps femoris } \\
\text { - Semitendinosus }\end{array}$ & $\begin{array}{l}\text { No significant differences in muscle activity between both } \\
\text { variations. } \\
\text { Major activation on the semitendinosus, vastus lateralis, } \\
\text { rectus femoris, and vastus lateralis, in this order. }\end{array}$ \\
\hline $\begin{array}{l}\text { (Paoli et al., } \\
\text { 2009) }\end{array}$ & $\begin{array}{l}6 \text { trained males } \\
\text { Age: } 25.8 \pm 3.7 \text { years } \\
\text { Height: } 182.0 \pm 3.5 \mathrm{~cm} \\
\text { Weight: } 83.2 \pm 5.8 \mathrm{~kg}\end{array}$ & - High bar squat & $\begin{array}{l}\text { 3S } 10 \mathrm{R}: \\
\text { 1) with no } \\
\text { weight } \\
\text { 2) } 30 \% \mathrm{RM} \\
\text { 3) } 70 \% \mathrm{RM} \\
\text { (3SW: } \\
\text { normal, x1.5 } \\
\& \times 2 \text { ) }\end{array}$ & $\begin{array}{l}\text { - Gluteus maximus } \\
\text { - Gluteus medialis } \\
\text { - Hip adductor } \\
\text { - Vastus medialis } \\
\text { - Vastus lateralis } \\
\text { - Rectus femoris } \\
\text { - Biceps femoris } \\
\text { - Semitendinosus }\end{array}$ & $\begin{array}{l}\text { Major activation was observed in vastus medialis, vastus } \\
\text { lateralis, and rectus femoris in the } 3 \text { stance widths. }\end{array}$ \\
\hline $\begin{array}{l}\text { (Caterisano et } \\
\text { al., 2002) }\end{array}$ & $\begin{array}{l}10 \text { males } \\
\text { Age: } 24.3 \pm 5.6 \text { years } \\
\text { Height: } 182.6 \pm 6.9 \mathrm{~cm} \\
\text { Weight: } 86.1 \pm 11.2 \mathrm{~kg} \\
\text { Body fat: } 6.1 \pm 1.8 \% \\
\text { Minimum experience: } 5 \\
\text { years }\end{array}$ & $\begin{array}{l}\text { - High-bar partial } \\
\text { squat } \\
\text { - High-bar squat } \\
\text { to parallel } \\
\text { - Full ROM high- } \\
\text { bar squat }\end{array}$ & $\begin{array}{l}3 \mathrm{R} \text { at } \\
\text { between } \\
100-125 \% \text { of } \\
\text { body-weight }\end{array}$ & $\begin{array}{l}\text { - Gluteus maximus } \\
\text { - Vastus medialis } \\
\text { - Vastus lateralis } \\
\text { - Biceps femoris }\end{array}$ & $\begin{array}{l}\text { - High-bar partial squat: Major activation on the vastus } \\
\text { medialis and lateralis. } \\
\text { - High-bar squat to parallel: Major activity on the vastus } \\
\text { medialis and lateralis, with higher activity on the gluteus } \\
\text { compared to partial. } \\
\text { - Full ROM high-bar squat: Major activation on the gluteus } \\
\text { in the concentric phase, and on the vastus medialis and } \\
\text { lateralis in the eccentric. }\end{array}$ \\
\hline $\begin{array}{l}\text { (Escamilla et al., } \\
\text { 2001) }\end{array}$ & $\begin{array}{l}10 \text { males } \\
\text { Age: } 29.6 \pm 6.5 \text { years } \\
\text { Height: } 177.0 \pm 8.5 \mathrm{~cm} \\
\text { Weight: } 93.5 \pm 14.0 \mathrm{~kg} \\
\text { Minimum experience: } \\
10.1 \pm 7.7 \text { years }\end{array}$ & $\begin{array}{l}\text { - High-bar squat } \\
\text { to parallel } \\
\text { Stance width: } \\
\text { narrow and wide. }\end{array}$ & 12RM & $\begin{array}{l}\text { - Vastus medialis } \\
\text { - Vastus lateralis } \\
\text { - Rectus femoris } \\
\text { - Semitendinosus } \\
\text { - Semimembranosus } \\
\text { - Gastrocnemius } \\
\end{array}$ & $\begin{array}{l}\text { Major activation on the vastus medialis, vastus lateralis, } \\
\text { and rectus femoris, in this order. No statistical difference } \\
\text { between these muscles. }\end{array}$ \\
\hline
\end{tabular}




\begin{tabular}{|c|c|c|c|c|c|}
\hline $\begin{array}{l}\text { (Boyden et al., } \\
\text { 2000) }\end{array}$ & $\begin{array}{l}6 \text { males } \\
\text { Age: } 23.0 \pm 4.1 \text { years } \\
\text { Height: } 180.0 \pm 3.0 \mathrm{~cm} \\
\text { Weight: } 80.95 \pm 1.5 \mathrm{~kg}\end{array}$ & $\begin{array}{l}\text { - High-bar squat } \\
\text { to parallel (four } \\
\text { different hip } \\
\text { rotations) }\end{array}$ & $\begin{array}{l}3 \mathrm{R} \text { at } 65 \% \\
\text { and } \\
75 \% 1 \mathrm{RM}\end{array}$ & $\begin{array}{l}\text { - Vastus medialis } \\
\text { - Vastus lateralis } \\
\text { - Rectus femoris }\end{array}$ & $\begin{array}{l}\text { Major activation at } 75 \% 1 \text { RM. No significant differences } \\
\text { between the hip rotations. }\end{array}$ \\
\hline $\begin{array}{l}\text { (Wright et al., } \\
\text { 1999) }\end{array}$ & $\begin{array}{l}6 \text { football players and } 5 \\
\text { bodybuilders }\end{array}$ & $\begin{array}{l}\text { - Full ROM high- } \\
\text { bar squat }\end{array}$ & $\begin{array}{l}3 \mathrm{R} \text { at } \\
75 \% 1 \mathrm{RM}\end{array}$ & $\begin{array}{l}\text { - Biceps femoris } \\
\text { - Semitendinosus }\end{array}$ & No statistical difference between both muscles. \\
\hline (Blanpied, 1999) & $\begin{array}{l}20 \text { females } \\
\text { Age: } 31.3 \pm 6.9 \text { years } \\
\text { Height: } 160.9 \pm 4.1 \mathrm{~cm} \\
\text { Weight: } 58.1 \pm 8.7 \mathrm{~kg}\end{array}$ & $\begin{array}{l}\text { - Guided squat } \\
\text { on Smith } \\
\text { machine }\end{array}$ & $\begin{array}{l}5 R \text {, feet in } \\
\text { line with the } \\
\text { body (IL) and } \\
\text { placed } \\
\text { forward (FF) }\end{array}$ & $\begin{array}{l}\text { - Gluteus maximus } \\
\text { - Vastus medialis } \\
\text { - Vastus lateralis } \\
\text { - Rectus femoris } \\
\text { - Biceps femoris } \\
\text { - Semitendinosus }\end{array}$ & $\begin{array}{l}\text { - FF: Major activation on the gluteus and biceps femoris, } \\
\text { semitendinosus, and semimembranosus. } \\
\text { - IL: Major activation on the gluteus and vastus medialis, } \\
\text { vastus lateralis, and rectus femoris. }\end{array}$ \\
\hline $\begin{array}{l}\text { (McCaw \& } \\
\text { Melrose, 1999) }\end{array}$ & $\begin{array}{l}9 \text { males } \\
\text { Age: } 22.0 \pm 1.0 \text { years } \\
\text { Height: } 183.0 \pm 8.0 \mathrm{~cm} \\
\text { Weight: } 92.0 \pm 14.0 \mathrm{~kg} \\
\text { Minimum experience: } \\
7.0 \pm 2.0 \text { years }\end{array}$ & - Low-bar squat & $\begin{array}{l}5 \mathrm{R} \text { at } 60- \\
75 \% 1 \mathrm{RM}, \\
\text { SW: closed, } \\
\text { normal, and } \\
\text { open }\end{array}$ & $\begin{array}{l}\text { - Gluteus maximus } \\
\text { - Hip adductor } \\
\text { - Vastus medialis } \\
\text { - Vastus lateralis } \\
\text { - Rectus femoris } \\
\text { - Biceps femoris } \\
\end{array}$ & $\begin{array}{l}\text { Major activation on the vastus lateralis, vastus medialis, } \\
\text { rectus femoris, and adductor longus with increasing load. } \\
\text { Major activation on the gluteus with increasing foot-width. }\end{array}$ \\
\hline
\end{tabular}

Age, height, weight, and minimum experience values are presented as Mean \pm Standard Deviation. ROM: Range of Motion; S: Sets; R: Repetition/s; RM: Repetition/s Maximum; SW: Stance Width; IL: feet in-line with the boy: FF: Forward placed Feet.

Table 2. Electromyographic activity reported in each study.

\begin{tabular}{|c|c|c|c|c|c|c|c|c|c|c|c|c|c|}
\hline \multirow{2}{*}{$\begin{array}{l}\text { (Author, } \\
\text { year) }\end{array}$} & \multirow{2}{*}{$\begin{array}{l}\text { EMG } \\
\text { value }\end{array}$} & \multirow[t]{2}{*}{ Squat } & \multicolumn{11}{|c|}{ Measured muscles } \\
\hline & & & GM & GMed & $\mathrm{HA}$ & $\mathrm{VL}$ & VM & RF & $\mathrm{BF}$ & ST & TA & GN & SL \\
\hline \multirow[t]{2}{*}{$\begin{array}{l}\text { (Bautista, } \\
\text { 2019) }\end{array}$} & $\begin{array}{l}\% \\
\text { IMCV }\end{array}$ & $\begin{array}{l}\text { Front } \\
\text { squat }\end{array}$ & - & - & - & $69.6 \pm 4.5$ & - & - & $24.3 \pm 5.1$ & - & - & - & - \\
\hline & & $\begin{array}{l}\text { Overhead } \\
\text { squat }\end{array}$ & - & - & - & $67.6 \pm 7.8$ & - & - & $24.3 \pm 5.1$ & - & - & - & - \\
\hline $\begin{array}{l}\text { (Delgado et } \\
\text { al., 2019) }\end{array}$ & $\begin{array}{l}\text { Raw } \\
(\mu \mathrm{V})\end{array}$ & $\begin{array}{l}\text { High-bar } \\
\text { squat (F) }\end{array}$ & $\sim 145$ & - & - & $\sim 350$ & - & - & $\sim 160$ & - & - & - & - \\
\hline $\begin{array}{l}\text { (Wu et al., } \\
\text { 2019) }\end{array}$ & $\begin{array}{l}\% \\
\text { MVC }\end{array}$ & $\begin{array}{l}\text { High-bar } \\
\text { squat }(P)\end{array}$ & - & - & - & $70.2 \pm 24.8$ & $66.4 \pm 24.4$ & $39.5 \pm 15.7$ & $12.9 \pm 14.9$ & $11.8 \pm 5.3$ & $30.5 \pm 11.1$ & $9.8 \pm 5.1$ & - \\
\hline \multirow[t]{2}{*}{$\begin{array}{l}\text { (Korak et al., } \\
2018)\end{array}$} & $\begin{array}{l}\% \\
\text { MVC }\end{array}$ & $\begin{array}{l}\text { High-bar } \\
\text { squat }(P)\end{array}$ & $\sim 80$ & - & - & $\sim 97$ & $\sim 96$ & $\sim 102$ & $\sim 78$ & - & - & - & - \\
\hline & & $\begin{array}{l}\text { Front } \\
\text { squat }\end{array}$ & $\sim 94$ & - & - & $\sim 102$ & $\sim 98$ & $\sim 101$ & $\sim 81$ & - & - & - & - \\
\hline $\begin{array}{l}\text { (Eliassen et } \\
\text { al., 2018) }\end{array}$ & $\begin{array}{l}\text { Raw } \\
(\mu \mathrm{V})\end{array}$ & $\begin{array}{l}\text { High-bar } \\
\text { squat (Q) }\end{array}$ & $130.5 \pm 25.0$ & $120.5 \pm 12.5$ & - & $308 \pm 33$ & $260 \pm 36$ & $231.5 \pm 34$ & $92.5 \pm 14.8$ & $70.5 \pm 11.0$ & - & $61.5 \pm 11.5$ & $88.0 \pm 11.0$ \\
\hline
\end{tabular}




\begin{tabular}{|c|c|c|c|c|c|c|c|c|c|c|c|c|c|}
\hline $\begin{array}{l}\text { (McCurdy et } \\
\text { al., 2018) }\end{array}$ & $\begin{array}{l}\% \\
\text { IMVC }\end{array}$ & $\begin{array}{l}\text { High-bar } \\
\text { squat }(P)\end{array}$ & $41.5 \pm 18.4$ & - & - & - & - & - & \multicolumn{2}{|c|}{ Hamstrings: $24.5 \pm 9.6$} & - & - & - \\
\hline $\begin{array}{l}\text { (Iversen et } \\
\text { al., 2017) }\end{array}$ & $\begin{array}{l}\% \\
\text { MVC }\end{array}$ & $\begin{array}{l}\text { High-bar } \\
\text { squat }(P)\end{array}$ & $\sim 33$ & - & - & $\sim 55$ & $\sim 65$ & $\sim 39$ & $\sim 14$ & $\sim 15$ & - & - & - \\
\hline \multirow[t]{2}{*}{$\begin{array}{l}\text { (da Silva et } \\
\text { al., 2017) }\end{array}$} & \multirow[t]{2}{*}{$\begin{array}{l}\% \\
\text { IMVC }\end{array}$} & $\begin{array}{l}\text { High-bar } \\
\text { squat }(P)\end{array}$ & $\sim 20$ & - & - & $\sim 60$ & $\sim 48$ & $\sim 60$ & $\sim 35$ & $\sim 30 \%$ & - & - & $\sim 30$ \\
\hline & & $\begin{array}{l}\text { High-bar } \\
\text { squat }(F)\end{array}$ & $\sim 15$ & - & - & $\sim 58$ & $\sim 48$ & $\sim 70$ & $\sim 30$ & $\sim 35$ & - & - & $\sim 25$ \\
\hline \multirow[t]{3}{*}{$\begin{array}{l}\text { (Contreras et } \\
\text { al., 2016) }\end{array}$} & \multirow[t]{3}{*}{$\begin{array}{l}\% \\
\text { IMVC }\end{array}$} & $\begin{array}{l}\text { High-bar } \\
\text { squat }(P)\end{array}$ & $37.5 \pm 20.0$ & - & - & $110.3 \pm 47.2$ & - & - & $14.9 \pm 6.6$ & - & - & - & - \\
\hline & & $\begin{array}{l}\text { High-bar } \\
\text { squat }(F)\end{array}$ & $35.9 \pm 18.9$ & - & - & $123.8 \pm 67.4$ & - & - & $14.4 \pm 6.4$ & - & - & - & - \\
\hline & & $\begin{array}{l}\text { Front } \\
\text { squat }\end{array}$ & $36.6 \pm 17.6$ & - & - & $124.2 \pm 72.9$ & - & - & $13.1 \pm 4.7$ & - & - & - & - \\
\hline \multirow[t]{3}{*}{$\begin{array}{l}\text { (Hammond } \\
\text { et al., 2016) }\end{array}$} & \multirow[t]{3}{*}{$\begin{array}{l}\% \\
\text { IMVC }\end{array}$} & $\begin{array}{l}\text { High-bar } \\
\text { squat (Q) }\end{array}$ & $\sim 15$ & - & - & $\sim 34$ & $\sim 39$ & - & $\sim 9$ & - & - & - & - \\
\hline & & $\begin{array}{l}\text { High-bar } \\
\text { squat }(P)\end{array}$ & $\sim 18$ & - & - & $\sim 37$ & $\sim 48$ & - & $\sim 14$ & - & - & - & - \\
\hline & & $\begin{array}{l}\text { High-bar } \\
\text { squat }(F)\end{array}$ & $\sim 15$ & - & - & $\sim 35$ & $\sim 48$ & - & $\sim 13$ & - & - & - & - \\
\hline $\begin{array}{l}\text { (Contreras et } \\
\text { al., 2015) }\end{array}$ & $\begin{array}{l}\% \\
\text { IMVC }\end{array}$ & $\begin{array}{l}\text { High-bar } \\
\text { squat }(P)\end{array}$ & $37.4 \pm 20.0$ & - & - & $110.4 \pm 47.2$ & - & - & $14.9 \pm 6.6$ & - & - & - & - \\
\hline \multirow[t]{2}{*}{$\begin{array}{l}\text { (Yavuz et al., } \\
2015)\end{array}$} & \multirow[t]{2}{*}{$\begin{array}{l}\% \\
\text { IMVC }\end{array}$} & $\begin{array}{l}\text { High-bar } \\
\text { squat }(P)\end{array}$ & $37.1 \pm 23.5$ & - & - & $47.0 \pm 15.1$ & $48.8 \pm 14$ & $36.7 \pm 12.4$ & $26.2 \pm 16.1$ & $21.5 \pm 12.0$ & - & - & - \\
\hline & & $\begin{array}{l}\text { Front } \\
\text { squat }\end{array}$ & $37.2 \pm 27.0$ & - & - & $51.2 \pm 17.3$ & $55.4 \pm 18$ & $46.1 \pm 21.7$ & $24.1 \pm 25.4$ & $16.0 \pm 9.0$ & - & - & - \\
\hline \multirow[t]{2}{*}{$\begin{array}{l}\text { (Aspe \& } \\
\text { Swinton, } \\
2014 \text { ) }\end{array}$} & \multirow[t]{2}{*}{$\begin{array}{l}\% \\
\text { MVC }\end{array}$} & $\begin{array}{l}\text { High-bar } \\
\text { squat (F) }\end{array}$ & $\begin{array}{c}60 \% \text { RM: } \\
43.2 \pm 27.1 \\
75 \% \text { RM: } \\
54.9 \pm 28.7 \\
90 \% \text { RM: } \\
58.4 \pm 31.1\end{array}$ & - & - & $\begin{array}{c}60 \% \mathrm{RM}: \\
69.1 \pm 22.7 \\
75 \% \mathrm{RM}: \\
75.3 \pm 25.3 \\
90 \% \mathrm{RM}: \\
79.1 \pm 55.6\end{array}$ & - & - & $\begin{array}{c}60 \% \mathrm{RM}: \\
42.5 \pm 20.8 \\
75 \% \mathrm{RM}: \\
49.8 \pm 24.6 \\
90 \% \mathrm{RM}: \\
52.1 \pm 24.9\end{array}$ & - & - & $\begin{array}{c}60 \% \text { RM: } \\
35.4 \pm 18.7 \\
75 \% \text { RM: } \\
44.0 \pm 28.6 \\
90 \% \text { RM: } \\
47.0 \pm 29.9\end{array}$ & - \\
\hline & & $\begin{array}{l}\text { Overhead } \\
\text { squat }\end{array}$ & $\begin{array}{c}60 \% \text { RM: } \\
31.7 \pm 17.7 \\
75 \% \text { RM: } \\
40.2 \pm 20.5 \\
90 \% \text { RM: } \\
39.8 \pm 21.1\end{array}$ & - & - & $\begin{array}{c}60 \% \mathrm{RM}: \\
62.2 \pm 21.4 \\
75 \% \mathrm{RM}: \\
68.0 \pm 22.4 \\
90 \% \mathrm{RM}: \\
70.4 \pm 23.1\end{array}$ & - & - & $\begin{array}{c}60 \% \mathrm{RM}: \\
36.2 \pm 20 \\
75 \% \mathrm{RM}: \\
42.1 \pm 20.6 \\
90 \% \mathrm{RM}: \\
44.5 \pm 26.2\end{array}$ & - & - & $\begin{array}{c}60 \% \text { RM: } \\
36.4 \pm 20.5 \\
75 \% \text { RM: } \\
34.8 \pm 16.6 \\
\\
90 \% \text { RM: } \\
36.4 \pm 14.7\end{array}$ & - \\
\hline
\end{tabular}




\begin{tabular}{|c|c|c|c|c|c|c|c|c|c|c|c|c|c|}
\hline $\begin{array}{l}\text { (Van den } \\
\text { Tillaar et al., } \\
\text { 2014) }\end{array}$ & $\begin{array}{l}\text { RMS } \\
(\mu \mathrm{V})\end{array}$ & $\begin{array}{l}\text { High-bar } \\
\text { squat }(P)\end{array}$ & - & - & - & $\sim 610$ & $\sim 600$ & $\sim 400$ & $\sim 180$ & - & - & - & - \\
\hline $\begin{array}{l}\text { (Andersen et } \\
\text { al., 2014) }\end{array}$ & $\begin{array}{l}\% \\
\text { IMVC }\end{array}$ & $\begin{array}{l}\text { High-bar } \\
\text { squat }(P)\end{array}$ & - & - & - & $\sim 80$ & $\sim 80$ & $\sim 90$ & $\sim 110$ & - & - & - & $\sim 95$ \\
\hline \multirow[t]{2}{*}{$\begin{array}{l}\text { (Gorsuch et } \\
\text { al., 2013) }\end{array}$} & $\begin{array}{l}\text { Raw } \\
(\mu \mathrm{V})\end{array}$ & $\begin{array}{l}\text { High-bar } \\
\text { squat }(P)\end{array}$ & - & - & - & - & - & $\sim 180$ & $\sim 180$ & - & - & $\sim 50$ & - \\
\hline & & $\begin{array}{l}\text { High-bar } \\
\text { squat (Q) }\end{array}$ & - & - & - & - & - & $\sim 140$ & $\sim 70$ & - & - & $\sim 50$ & - \\
\hline $\begin{array}{l}\text { (Lynn \& } \\
\text { Noffal, 2012) }\end{array}$ & $\begin{array}{l}\% \\
\text { MVC } \\
\end{array}$ & $\begin{array}{l}\text { High-bar } \\
\text { squat }(F)\end{array}$ & $20 \pm 14.2$ & - & - & - & - & $64.4 \pm 44.5$ & $20.6 \pm 21.9$ & - & - & - & - \\
\hline $\begin{array}{l}\text { (Robbins, } \\
\text { 2011) }\end{array}$ & $\begin{array}{l}\% \\
\text { MVC }\end{array}$ & $\begin{array}{l}\text { High-bar } \\
\text { squat }(P)\end{array}$ & $\sim 38$ & - & - & - & $\sim 81$ & - & $\sim 23$ & - & - & $\sim 40$ & - \\
\hline $\begin{array}{l}\text { (Pereira et } \\
\text { al., 2010) }\end{array}$ & $\begin{array}{l}\text { \% } \\
\text { IMVC }\end{array}$ & $\begin{array}{l}\text { High-bar } \\
\text { squat }(P)\end{array}$ & - & - & $\begin{array}{l}\text { HER } 0^{\circ}: \\
\sim 14 \\
\text { HER } 30^{\circ}: \\
\sim 20 \\
\text { HER 50\%: } \\
\sim 20\end{array}$ & - & - & $\begin{array}{l}\text { HER } 0^{\circ}: \\
\sim 41 \\
\text { HER } 30^{\circ}: \\
\sim 47 \\
\text { HER } 50^{\circ}: \\
\sim 41\end{array}$ & - & - & - & - & - \\
\hline $\begin{array}{l}\text { (Fauth et al., } \\
2010)\end{array}$ & $\begin{array}{l}\% \\
\text { IMVC }\end{array}$ & $\begin{array}{l}\text { High-bar } \\
\text { squat (F) }\end{array}$ & $90 \pm 42$ & $26 \pm 13$ & - & $114 \pm 54$ & $133 \pm 52$ & $81 \pm 35$ & $45 \pm 20$ & $37 \pm 25$ & - & - & - \\
\hline $\begin{array}{l}\text { (Ebben et al., } \\
2009 \text { ) }\end{array}$ & $\begin{array}{l}\% \\
\text { IMVC }\end{array}$ & $\begin{array}{l}\text { High-bar } \\
\text { squat }(F)\end{array}$ & - & - & - & $\sim 90$ & - & $\sim 78$ & $\sim 37$ & - & - & - & - \\
\hline \multirow[t]{2}{*}{$\begin{array}{l}\text { (Schwanbeck } \\
\text { et al., 2009) }\end{array}$} & $\begin{array}{l}\% \\
\text { MVC }\end{array}$ & $\begin{array}{l}\text { Guided } \\
\text { squat }\end{array}$ & - & - & - & $\sim 60$ & $\sim 60$ & - & $\sim 18$ & - & $\sim 30$ & $\sim 20$ & - \\
\hline & & $\begin{array}{l}\text { High-bar } \\
\text { squat }(P)\end{array}$ & - & - & - & $\sim 80$ & $\sim 81$ & - & $\sim 20$ & - & $\sim 59$ & $\sim 30$ & - \\
\hline \multirow[t]{2}{*}{$\begin{array}{l}\text { (Gullett et al., } \\
2009 \text { ) }\end{array}$} & $\begin{array}{l}\% \\
\text { IMVC }\end{array}$ & $\begin{array}{l}\text { High-bar } \\
\text { squat }(P)\end{array}$ & - & - & - & $\sim 61$ & $\sim 80$ & $\begin{array}{l}\sim 62 \\
\end{array}$ & $\sim 20$ & $\sim 130$ & - & - & - \\
\hline & & $\begin{array}{l}\text { Front } \\
\text { squat }\end{array}$ & - & - & - & $\sim 60$ & $\sim 81$ & $\sim 59$ & $\sim 19$ & $\sim 140$ & - & - & - \\
\hline $\begin{array}{l}\text { (Paoli et al., } \\
2009)\end{array}$ & $\begin{array}{l}\text { RMS } \\
\text { RMS }\end{array}$ & $\begin{array}{l}\text { High-bar } \\
\text { squat }(P)\end{array}$ & $\begin{array}{c}\text { NW: } \\
20.5 \pm 5 \\
x 1.5: \\
24.1 \pm 9 \\
x 2: \\
28.8 \pm 7\end{array}$ & $\begin{array}{c}\text { NW: } \\
25.2 \pm 7 \\
x 1.5: \\
26.5 \pm 7 \\
x 2: \\
31.8 \pm 12\end{array}$ & $\begin{array}{c}\text { NW: } \\
17.0 \pm 5 \\
\text { x1.5: } \\
16.6 \pm 7 \\
\text { x2: } \\
16.9 \pm 6\end{array}$ & $\begin{array}{c}\text { NW: } \\
60.5 \pm 7 \\
\text { x1.5: } \\
59.7 \pm 9 \\
\text { x2: } \\
66.0 \pm 11\end{array}$ & $\begin{array}{c}\text { NW: } \\
57.3 \pm 7 \\
\text { x1.5: } \\
57.5 \pm 9 \\
\text { x2: } \\
53.2 \pm 15\end{array}$ & $\begin{array}{c}\text { NW: } \\
57.1 \pm 8 \\
x 1.5: \\
50.4 \pm 8 \\
\text { x2: } \\
51.6 \pm 0.1\end{array}$ & $\begin{array}{c}\text { NW: } \\
24.5 \pm 3 \\
x 1.5: \\
25.6 \pm 6 \\
x 2: \\
27.2 \pm 7\end{array}$ & $\begin{array}{c}\text { NW: } \\
23.2 \pm 7 \\
\text { x1.5: } \\
23.8 \pm 10 \\
\text { x2: } \\
25.4 \pm 11\end{array}$ & - & - & - \\
\hline $\begin{array}{l}\text { (Caterisano } \\
\text { et al., 2002) } \\
\end{array}$ & $\begin{array}{l}\% \\
\text { MVC }\end{array}$ & $\begin{array}{l}\text { High-bar } \\
\text { squat (Q) }\end{array}$ & $\sim 15$ & - & - & $38.6 \pm 12.3$ & $35.4 \pm 13.3$ & - & $\sim 11$ & - & - & - & - \\
\hline
\end{tabular}

\section{VOLUME 15 | Proc4 | 2020 | S1289}




\begin{tabular}{|c|c|c|c|c|c|c|c|c|c|c|c|c|c|}
\hline & & $\begin{array}{l}\text { High-bar } \\
\text { squat }(P)\end{array}$ & $\sim 19$ & - & - & $38.4 \pm 12.9$ & $31.1 \pm 9.7$ & - & $\sim 11$ & - & - & - & - \\
\hline & & $\begin{array}{l}\text { High-bar } \\
\text { squat }(F)\end{array}$ & $\sim 24$ & - & - & $32 \pm 10.5$ & $31.7 \pm 10.3$ & - & $\sim 12$ & - & - & - & - \\
\hline $\begin{array}{l}\text { (Escamilla et } \\
\text { al., 2001) }\end{array}$ & $\begin{array}{l}\% \\
\text { IMVC }\end{array}$ & $\begin{array}{l}\text { High-bar } \\
\text { squat }(P)\end{array}$ & - & - & - & $\begin{array}{l}\text { NS: } 39.5 \pm 7 \\
\text { WS: } 40 \pm 7\end{array}$ & $\begin{array}{c}\text { NS: } 42 \pm 8 \\
\text { WS:41.5 } \pm 5\end{array}$ & $\begin{array}{c}\text { NS: } \\
32 \pm 13.5 \\
\text { WS: } \\
8.5 \pm 11\end{array}$ & $\begin{array}{c}\text { NS: } 18 \pm 8 \\
\text { WS: } \\
19 \pm 8.5\end{array}$ & $\begin{array}{c}\text { NS: } 16 \pm 7 \\
\text { WS: } \\
18.5 \pm 8\end{array}$ & - & $\begin{array}{l}\text { NS: } 14.5 \pm 4 \\
\text { WS: } 13.5 \pm 5\end{array}$ & - \\
\hline $\begin{array}{l}\text { (Boyden et } \\
\text { al., 2000) }\end{array}$ & $\begin{array}{l}\% \\
\text { MVC }\end{array}$ & $\begin{array}{l}\text { High-bar } \\
\text { squat }(P)\end{array}$ & - & - & - & $\begin{array}{c}\text { HIR 10०: } \\
65 \%: \\
95.3 \pm 1.2 \\
75 \%: \\
95.8 \pm 1.2 \\
0^{\circ}: \\
65 \%: \\
94.2 \pm 1.2 \\
75 \%: \\
95.2 \pm 1.0 \\
\text { HER } 10^{\circ}: \\
65 \%: \\
93.8 \pm 1.0 \\
75 \%: \\
96.2 \pm 1.0 \\
\text { HER } 20^{\circ}: \\
65 \%: \\
94.6 \pm 2.1 \\
75 \%: \\
97.1 \pm 0.8\end{array}$ & $\begin{array}{c}\text { HIR 10०: } \\
65 \%: \\
98.1 \pm 0.7 \\
75 \%: \\
96.9 \pm 1.2 \\
0^{\circ}: \\
65 \%: \\
98.6 \pm 0.5 . \\
75 \%: \\
97.7 \pm 0.7 \\
\text { HER } 10^{\circ}: \\
65 \%: \\
97.6 \pm 0.7 . \\
75 \%: \\
97.9 \pm 0.8 \\
\text { HER } 20{ }^{\circ}: \\
65 \%: \\
96.5 \pm 1.1 \\
75 \%: \\
96.9 \pm 1.2\end{array}$ & $\begin{array}{c}\text { HIR 10०: } \\
65 \%: \\
88.8 \pm 2 \\
75 \%: \\
92.2 \pm 2 \\
0^{\circ}: \\
65 \%: \\
83.8 \pm 2 \\
75 \%: \\
89.5 \pm 2 \\
\text { HER } 10^{\circ}: \\
65 \%: \\
89.0 \pm 3 \\
75 \%: \\
93.2 \pm 1 \\
\text { HER } 20^{\circ}: \\
65 \%: \\
86.9 \pm 4 \\
75 \%: \\
92.1 \pm 2\end{array}$ & - & - & - & - & - \\
\hline $\begin{array}{l}\text { (Wright et al., } \\
\text { 1999) }\end{array}$ & $\begin{array}{l}\% \\
\text { MVC }\end{array}$ & $\begin{array}{l}\text { High-bar } \\
\text { squat (F) }\end{array}$ & - & - & - & - & - & - & $43.5 \pm 13.3$ & $45.9 \pm 13.7$ & - & - & - \\
\hline $\begin{array}{l}\text { (Blanpied, } \\
\text { 1999) }\end{array}$ & $\begin{array}{l}\% \\
\text { IMVC }\end{array}$ & $\begin{array}{l}\text { Guided } \\
\text { squat }\end{array}$ & - & - & - & $\begin{array}{c}\mathrm{IL}: \\
20.3 \pm 12.5 \\
\mathrm{FF}: \\
21.1 \pm 13.1\end{array}$ & $\begin{array}{c}\mathrm{IL}: \\
12.5 \pm 5.6 \\
\mathrm{FF}: \\
26.6 \pm 9.9\end{array}$ & $\begin{array}{c}\mathrm{IL}: \\
24.5 \pm 8.8 \\
\mathrm{FF}: \\
30.5 \pm 9.3\end{array}$ & - & - & - & - & - \\
\hline
\end{tabular}




\begin{tabular}{|c|c|c|c|c|c|c|c|c|c|c|c|c|c|}
\hline $\begin{array}{l}\text { (McCaw \& } \\
\text { Melrose, } \\
1999)\end{array}$ & $\begin{array}{l}\text { Raw } \\
\left(\mu V^{*} s\right)\end{array}$ & $\begin{array}{l}\text { Low-bar } \\
\text { squat }\end{array}$ & $\begin{array}{c}60 \% \mathrm{RM}: \\
\text { (NS): } \\
5.2 \pm 2.8 \\
\text { (NW): } \\
5.0 \pm 2.8 \\
\text { (WS): } \\
5.3 \pm 3.9 \\
75 \% \mathrm{RM}: \\
\text { (NS): } \\
5.9 \pm 3.5 \\
\text { (NW): } \\
5.5 \pm 3.2 \\
\text { (WS): } \\
6.9 \pm 3.8\end{array}$ & - & $\begin{array}{c}60 \% \mathrm{RM}: \\
\text { (NS): } \\
6.1 \pm 1.9 \\
\text { (NW): } \\
2.85 \pm 1.75 \\
\text { (WS): } \\
2.7 \pm 1.9 \\
75 \% \mathrm{RM} \\
\text { (NS): } \\
3.7 \pm 2.6 \\
\text { (NW): } \\
3.7 \pm 2.3 \\
\text { (WS): } \\
3.8 \pm 2.4\end{array}$ & $\begin{array}{c}60 \% \mathrm{RM}: \\
\text { (NS): } \\
22.7 \pm 7.1 \\
\text { (NW): } \\
22.1 \pm 6.6 \\
\text { (WS): } \\
22.7 \pm 7.9 \\
75 \% \mathrm{RM}: \\
\text { (NS): } \\
26.7 \pm 8.4 \\
\text { (NW): } \\
27.1 \pm 8.9 \\
\text { (WS): } \\
27.1 \pm 8.7\end{array}$ & $\begin{array}{c}\text { 60\% RM: } \\
\text { (NS): } \\
19.5 \pm 10.6 \\
\text { (NW): } \\
19.2 \pm 9.9 \\
\text { (WS): } \\
19.9 \pm 10.7 \\
75 \% \text { RM: } \\
\text { (NS): } \\
23.4 \pm 12.4 \\
\text { (NW): } \\
22.7 \pm 12.4 \\
\text { (WS): } \\
22.6 \pm 12.7\end{array}$ & $\begin{array}{c}\text { 60\% RM: } \\
\text { (NS): } \\
11.05 \pm 7.4 \\
\text { (NW): } \\
10.6 \pm 7.0 \\
\text { (WS): } \\
11.1 \pm 8.1 \\
75 \% \text { RM: } \\
\text { (NS): } \\
13.1 \pm 8.7 \\
\text { (NW): } \\
13.2 \pm 8.5 \\
\text { (WS): } \\
14.0 \pm 9.4\end{array}$ & - & - & - & 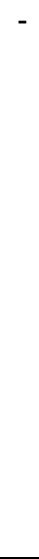 & - \\
\hline
\end{tabular}

Values are expressed as Mean \pm Standard Deviation or as a percentage. EMG: Electromyography; GM: Gluteus Maximus; GMed: Gluteus Medialis; HA: Hip Adductors; VL: Vastus Lateralis; VM: Vastus Medialis; RF: Rectus Femoris; BF: Biceps Femoris; ST: Semitendinosus; TA: Tibialis Anterior; GN: Gastrocnemius; SL: Soleus; \%IMCV: percentage of an Isometric Maximum Voluntary Contraction; \%MVC: percentage of the historic Maximum Voluntary Contraction; \%RMS: percentage of peak Root Mean Square (RMS); $\mu$ V: microvolts; F: full range of motion squat; P: squat to parallel; Q: quarter of a full ROM squat (partial squat); HIR/HER: Hip Internal/External Rotation; NW: Normal Width; X1.5: stance width at 1.5 times the normal stance; X2: stance width at 2 times the normal stance; NS: Narrow Stance; WS: Wide Stance; IL: feet in line with the body; FF: Feet placed Forward of the body line. 
observed a slightly higher activation on the gluteus in the full-ROM squats in comparison to the other two modalities, other authors found no differences, or even higher gluteus activity values in the parallel squats (da Silva et al., 2017; Hammond et al., 2016). The effect of this technical modification was only assessed in the high-bar squat.

\section{Stance width}

This technical modification was included in the analysis of the high-bar (Escamilla et al., 2001; Paoli et al., 2009) and the low-bar (McCaw \& Melrose, 1999) squat. The main effect of having a wider stance was a higher activation on the gluteus (McCaw \& Melrose, 1999; Paoli et al., 2009). Variations on the stance width did not produce an effect on the activation of the quadriceps, hamstring, and gastrocnemius (Escamilla et al., 2001).

\section{Hip rotation}

Different hip rotations (i.e. orientation plane of the foot or knees) were only tested in high-bar squats. The main results of this technical modification were an increase in the activity of the hip adductors (Pereira et al., 2010). No significant effects of different hip rotations on the quadriceps were observed (Boyden et al., 2000).

Feet placement in line with the body or ahead

This technical modification was only tested in the guided squat, as this machine allows the subject to place the feet in line with the body or ahead. When the feet were placed in line with the body major activity on the quadriceps (i.e. normal activity pattern of a high-bar squat) was observed. In turn, when the feet were placed in front of the body line, higher activity was detected on the gluteus and hamstrings compared to the quadriceps (Blanpied, 1999).

Table 3. Quality assessment of the included studies.

\begin{tabular}{|c|c|c|c|c|c|c|c|}
\hline Author (year) & 1 & 2 & 3 & 4 & 5 & 6 & Total \\
\hline Bautista (2019) & + & + & + & + & + & + & 6 \\
\hline Andersen (2019) & + & + & + & + & + & + & 6 \\
\hline Delgado (2019) & + & + & + & + & + & + & 6 \\
\hline Wu (2019) & + & + & + & + & + & + & 6 \\
\hline Korak (2018) & + & + & + & + & + & + & 6 \\
\hline Elliasen (2018) & + & + & + & + & + & + & 6 \\
\hline McCurdy (2018) & + & + & + & + & + & + & 6 \\
\hline Iversen (2017) & + & - & + & + & + & + & 5 \\
\hline da Silva (2017) & + & + & + & + & + & + & 6 \\
\hline Contreras (2016) & + & + & + & + & + & + & 6 \\
\hline Hammond (2016) & + & + & + & + & + & + & 6 \\
\hline Contreras (2015) & + & + & + & + & + & + & 6 \\
\hline Yavuz (2015) & + & + & + & + & + & + & 6 \\
\hline Aspe (2014) & + & + & + & + & + & + & 6 \\
\hline Van den Tillar (2014) & + & - & + & + & + & + & 5 \\
\hline Andersen (2014) & + & + & + & + & + & + & 6 \\
\hline Gorsuch (2013) & + & + & + & + & + & + & 6 \\
\hline Lynn (2012) & + & + & + & + & + & + & 6 \\
\hline Robbins (2011) & + & + & + & + & + & + & 6 \\
\hline Pereira (2010) & + & + & + & + & + & + & 6 \\
\hline Fauth (2010) & + & + & + & + & + & + & 6 \\
\hline
\end{tabular}




\begin{tabular}{|c|c|c|c|c|c|c|c|}
\hline Ebben (2009) & + & + & + & + & + & + & 6 \\
\hline Schwanbeck (2009) & + & + & + & + & + & + & 6 \\
\hline Gullett (2009) & + & + & + & + & + & + & 6 \\
\hline Paoli (2009) & + & + & + & + & + & + & 6 \\
\hline Caterisano (2002) & + & + & + & + & + & + & 6 \\
\hline Escamilla (2001) & + & + & + & + & + & + & 6 \\
\hline Boyden (2000) & + & + & + & + & + & + & 6 \\
\hline Wright (1999) & + & + & + & + & + & + & 6 \\
\hline Blanpied (1999) & + & + & + & + & + & + & 6 \\
\hline McCaw (1999) & + & + & + & + & + & + & 6 \\
\hline
\end{tabular}

\section{DISCUSSION}

This study aimed to gather data on the muscular activation of the lower limb during five different variations of the squat exercise (i.e. high-bar squat, front squat, overhead squat, guided squat, and low-bar squat), looking forward to identifying the main muscle group involved and the variation with the higher activation levels. In the following lines, the main findings are going to be compared and discussed following the scientific body of knowledge.

In summary, almost all the studies found the major activity on the anterior thigh muscles, which are involved in the knee extension and are part of the quadriceps (vastus lateralis, vastus medialis, and rectus femoris), with the highest activation observed on the vastus lateralis. Only Andersen et al. (2014) and Gullet et al. (2009), reported higher activation levels on the hamstrings than on the quadriceps, in the front squat and high-bar squat, respectively. These uneven results may be due to the secondary function of the hamstrings as hip extensors (Netter, 1999). Also, the hamstrings (biceps femoris, semitendinosus, semimembranosus) are not actual antagonists in the squat exercise, but contract with the quadriceps in their function of stabilizing the tibia and the knee joint (Schoenfeld, 2010). However, hamstrings activation should be only moderate during the squat performance (Escamilla et al., 2001). Regarding the comparison between the gluteus and hamstrings, there is some controversy on which has a higher activation (see "results: high-bar squat" section, and Table 2). Attending to the squat biomechanics, the gluteus act as a powerful hip extensor and also as a knee and hip stabilizer. Gluteus activation mainly depends on the force arm length which is conditioned by different technique factors such as the depth, the stance width (McCaw \& Melrose, 1999; Paoli et al., 2009; Schoenfeld, 2010). Concerning the calves, low activation levels have been observed in comparison to the thigh muscles. Attending to their main functions (Netter, 1999), these lower levels of activation may reside in the use of stable surfaces to perform the squat and the limited contribution of the ankle muscles in the squat movement.

As can be seen in the results, there are technical and electromyographical variations when the position of the bar changes (Pham et al., 2020). For instance, the load in the performance of a high-bar squat, a front squat, or an overhead squat, is shared between the knees and the hips (Comfort et al., 2018), with the main focus on the vastus lateralis and medialis as knee extensors (Aspe \& Swinton, 2014; Contreras et al., 2015, 2016; Delgado et al., 2019; Ebben et al., 2009; Hammond et al., 2016). In turn, a higher hip involvement has been reported in the low-bar squat (Glassbrook et al., 2017, 2019; Wretenberg et al., 1996). In this variation, the trunk inclination is greater, and thus, gluteus and hip extensors activity is enhanced in comparison to other variations of the squat exercise. However, the low-bar squat stills a knee-extensors dominant exercise (McCaw \& Melrose, 1999). Further research on the electromyographic activity of this squat variation is needed to better understand the neuromuscular processes involved. 


\section{Comparing the activation levels of each variation}

Firstly, it is important to bear in mind that higher levels of muscle activation are enhanced in the squat exercise with increasing loads (Aspe \& Swinton, 2014; Boyden et al., 2000; McCaw \& Melrose, 1999; Paoli et al., 2009). The lever arm between the external load (i.e. the barbell) and the centre of mass of the body plays an important role in this regard (Gullett et al., 2009).

In the low-bar squat the lever arm is relatively shorter, and the position of the bar (below the acromion) is more biomechanical favourable than in the rest of the variations (Glassbrook et al., 2017, 2019; Wretenberg et al., 1996; see figure 2). Due to these abovementioned facts, the low-bar squat is the variation in which higher loads can be used, and thus, higher activation levels may be achieved. No significant differences were observed between the activation in a high-bar squat and a front squat (Gullett et al., 2009; Korak et al., 2018) and thus, both would be classified at the same level after the low-bar squat. These authors reported similar knee extension momentum, and comparable gluteus implication has been reported in both variations of the squat exercise (Neto et al., 2020). Concerning the overhead squat, lower muscle activity on the lower limb has been observed in comparison to the aforementioned variations. This is due to the greater involvement of the upper body to hold the bar and stabilize the spine during the execution (Aspe \& Swinton, 2014; Bautista, 2019). Moreover, there are many factors such as the strength and shoulder mobility, which limit the load used in this exercise. Understanding that load increases entail increases in activation level (Aspe \& Swinton, 2014; Boyden et al., 2000; McCaw \& Melrose, 1999; Paoli et al., 2009), this squat variation would be positioned after the low-bar squat, the high-bar, and the front squat. Finally, Schwanbeck et al. (2009), Blanpied (1999), and Clark et al. (2012) in their revision of the literature pointed the guided squat as the variation provoking the lowest activation levels. One possible explanation for these results would be the nature of a guided exercise, eliminating most of the activity of the stabilizers.

\section{Technical factors involved}

Apart from the total load used, the depth has been shown to influence muscle activity patterns and the level of activation. In this line, lower activation levels were observed in partial squats (i.e. approximately $45^{\circ}$ of knee movement) compared to the parallel or full range of motion squats (Gorsuch et al., 2013; Hammond et al., 2016). Similarly, Paoli et al. (2010) observed in their study that a reduction in the ROM decreased muscle activation levels on the shoulder when performing a military press. This finding relates to ours and strengthens the idea that a greater ROM entails a greater muscle activation. In terms of the influence of the ROM on the muscle activity pattern, no significant conclusions could be extracted from the analyses, with contrary findings among the reviewed articles (Caterisano et al., 2002; da Silva et al., 2017; Hammond et al., 2016; Neto et al., 2020). The stance width is another parameter that influences the muscle activity patterns in the squat. For instance, the hamstrings, gluteus maximus, and the hip adductor have all shown significantly greater activity in the wider stance squat compared with the narrow stance (Escamilla et al., 2001; McCaw \& Melrose, 1999; Paoli et al., 2009). One of the main functions of the gluteus maximus is hip abduction (Netter, 1999) and thus, a wider stance facilitates this action of the gluteus. No other muscle activity was altered with varying stance widths (Escamilla et al., 2001). Finally, the rotation of the hips has been shown to increase the activation of the hip adductors (Pereira et al., 2010), with no significant changes in the activation patterns of the rest of the analysed muscles (Boyden et al., 2000).

\section{Limitations}

The included studies have some limitations that should be listed. In this regard, none of the studies indicate what type of isometric contraction (e.g. pushing or holding; Schaefer \& Bittmann, 2017) performed the subjects to obtain the isometric maximum voluntary contraction to standardize the results. In this line, it is 
worth mentioning that standardization values are uneven, and this may entail a problem when trying to compare and discuss results. Also, one study found significant differences between the upper and lower fibres of the gluteus (Contreras et al., 2016). These differences between fibre bundles of the same muscle may condition the EMG results and thus, measurement procedures should be clearly stated in future studies. Finally, and even all the procedures of the present review were carefully carried out it is not free of limitations. The standardization made in the values by the authors may limit the analysis of each phase of the execution (i.e. eccentric and concentric). Future studies should review the literature comparing the activation in each phase. Also, the disparities between the included studies may carry to limited comparisons and extraction of conclusions. Finally, our inclusion criteria did not include variable resistance or different bar-types. These factors may provide the strength and conditioning professionals and athletes to further understanding of squat exercises.

\section{CONCLUSION}

This study highlights the importance of studying the neuromuscular acute effects of the squat to deeply understand the exercise and its variations and individualize resistance exercise programs. In brief, we observed that the squat, independently of the variation performed, is a knee-extensor dominant exercise. Different variations entailed different activation pattern, and activation levels. The low bar squat was the variation with higher activation levels due to the possibility of using a higher load. This movement has a considerable involvement of the hip muscles. High-bar squat and front squat provoked similar activation patterns due to having a similar lever arm. In this regard, the lever arm is greater in the overhead squat and thus, the activation levels are lesser (due to a limited capacity of using a high load). Finally, the guided squat was the variation with lower activation levels due to not require stabilization. The evidence presented in this study may help the strength and conditioning professionals and practitioners with the exercise selection depending on the muscular targets and the individual characteristics of the athlete.

\section{REFERENCES}

Andersen, V., Fimland, M. S., Brennset, O., Haslestad, L. R., Lundteigen, M. S., Skalleberg, K., \& Saeterbakken, A. H. (2014). Muscle activation and strength in squat and Bulgarian squat on stable and unstable surface. International Journal of Sports Medicine, 35(14), 1196-1202. https://doi.org/10.1055/s-0034-1382016

Andersen, Vidar, Steiro Fimland, M., Knutson Kolnes, M., Jensen, S., Laume, M., \& Saeterbakken, A. H. (2016). Electromyographic comparison of squats using constant or variable resistance. Journal of Strength and Conditioning Research, 30(12), 3456-3463. https://doi.org/10.1519/JSC.0000000000001451

Aspe, R. R., \& Swinton, P. A. (2014). Electromyographic and kinetic comparison of the back squat and overhead squat. Journal of Strength and Conditioning Research, 28(10), 2827-2836. https://doi.org/10.1519/JSC.0000000000000462

Bautista, D. (2019). Electromyographic comparison of the front squat and overhead squat [Master Thesis]. California State University.

Blanpied, P. R. (1999). Changes in muscle activation during wall slides and squat-machine exercise. Journal of Sport Rehabilitation, 8(2), 123-134. https://doi.org/10.1123/jsr.8.2.123

Bolgla, L. A., \& Uhl, T. L. (2005). Electromyographic analysis of hip rehabilitation exercises in a group of healthy subjects. Journal of Orthopaedic \& Sports Physical Therapy, 35(8), 487-494. https://doi.org/10.2519/jospt.2005.35.8.487 
Borreani, S., Calatayud, J., Martin, J., Colado, J. C., Tella, V., \& Behm, D. (2014). Exercise intensity progression for exercises performed on unstable and stable platforms based on ankle muscle activation. Gait \& Posture, 39(1), 404-409. https://doi.org/10.1016/j.gaitpost.2013.08.006

Bourne, M. N., Duhig, S. J., Timmins, R. G., Williams, M. D., Opar, D. A., Al Najjar, A., Kerr, G. K., \& Shield, A. J. (2017). Impact of the Nordic hamstring and hip extension exercises on hamstring architecture and morphology: Implications for injury prevention. British Journal of Sports Medicine, 51(5), 469-477. https://doi.org/10.1136/bjsports-2016-096130

Boyden, G., Scurr, J., \& Dyson, R. (2000). A comparison of quadriceps electromyographic activity with the position of the foot during the parallel squat. Journal of Strength and Conditioning Research, 14(4), 379-382. https://doi.org/10.1519/00124278-200011000-00002

Caterisano, A., Moss, R. F., Pellinger, T. K., Woodruff, K., Lewis, V. C., Booth, W., \& Khadra, T. (2002). The effect of back squat depth on the EMG activity of 4 superficial hip and thigh muscles. Journal of Strength and Conditioning Research, 16(3), 428-432. https://doi.org/10.1519/00124278-20020800000014

Chowdhury, R. H., Reaz, M. B. I., Ali, M. A. B. M., Bakar, A. A. A., Chellappan, K., \& Chang, T. G. (2013). Surface electromyography signal processing and classification techniques. Sensors (Basel, Switzerland), 13(9), 12431-12466. https://doi.org/10.3390/s130912431

Clark, D. R., Lambert, M. I., \& Hunter, A. M. (2012). Muscle activation in the loaded free barbell squat: A brief review. Journal of Strength and Conditioning Research, 26(4), 1169-1178. https://doi.org/10.1519/JSC.0b013e31822d533d

Comfort, P., McMahon, J., \& Suchomel, T. (2018). Optimizing squat technique-Revisited. Strength and Conditioning Journal, 40(6), 68-74. https://doi.org/10.1519/SSC.0000000000000398

Contreras, B., Vigotsky, A. D., Schoenfeld, B. J., Beardsley, C., \& Cronin, J. (2015). A comparison of gluteus maximus, biceps femoris, and vastus lateralis electromyographic activity in the back squat and barbell hip thrust exercises. Journal of Applied Biomechanics, 31(6), 452-458. https://doi.org/10.1123/jab.2014-0301

Contreras, B., Vigotsky, A. D., Schoenfeld, B. J., Beardsley, C., \& Cronin, J. (2016). A comparison of gluteus maximus, biceps femoris, and vastus lateralis electromyography amplitude in the parallel, full, and front squat variations in resistance-trained females. Journal of Applied Biomechanics, 32(1), 16-22. https://doi.org/10.1123/jab.2015-0113

da Silva, J. J., Schoenfeld, B. J., Marchetti, P. N., Pecoraro, S. L., Greve, J. M. D., \& Marchetti, P. H. (2017). Muscle activation differs between partial and full back squat exercise with external load equated. Journal of Strength and Conditioning Research, 31(6), 1688-1693. https://doi.org/10.1519/JSC.0000000000001713

Delgado, J., Drinkwater, E. J., Banyard, H. G., Haff, G. G., \& Nosaka, K. (2019). Comparison between back squat, romanian deadlift, and barbell hip thrust for leg and hip muscle activities during hip extension. Journal of Strength and Conditioning Research, 33(10), 2595-2601. https://doi.org/10.1519/JSC.0000000000003290

Ebben, W. P., Feldmann, C. R., Dayne, A., Mitsche, D., Alexander, P., \& Knetzger, K. J. (2009). Muscle activation during lower body resistance training. International Journal of Sports Medicine, 30(1), 1-8. https://doi.org/10.1055/s-2008-1038785

Eliassen, W., Saeterbakken, A. H., \& van den Tillaar, R. (2018). Comparison of bilateral and unilateral squat exercises on barbell kinematics and muscle activation. International Journal of Sports Physical Therapy, 13(5), 871-881. https://doi.org/10.26603/ijspt20180871

Escamilla, R. F., Fleisig, G. S., Lowry, T. M., Barrentine, S. W., \& Andrews, J. R. (2001). A threedimensional biomechanical analysis of the squat during varying stance widths. Medicine and Science in Sports and Exercise, 33(6), 984-998. https://doi.org/10.1097/00005768-200106000-00019 
Fauth, M. L., Garceau, L. R., Lutsch, B., Gray, A., Szalkowski, C., Wurm, B., \& Ebben, W. P. (2010). Hamstrings, quadriceps, and gluteal muscle activation during resistance training exercises. Proceedings of the 28th Conference of the International Society of Biomechanics in Sports, 195-198.

Glassbrook, D. J., Brown, S. R., Helms, E. R., Duncan, S., \& Storey, A. G. (2019). The high-bar and lowbar back-squats: A biomechanical analysis. Journal of Strength and Conditioning Research, 33 Suppl 1, S1-S18. https://doi.org/10.1519/JSC.0000000000001836

Glassbrook, D. J., Helms, E. R., Brown, S. R., \& Storey, A. G. (2017). A review of the biomechanical differences between the high-bar and low-bar back-squat. Journal of Strength and Conditioning Research, 31(9), 2618-2634. https://doi.org/10.1519/JSC.0000000000002007

Gorsuch, J., Long, J., Miller, K., Primeau, K., Rutledge, S., Sossong, A., \& Durocher, J. J. (2013). The effect of squat depth on multiarticular muscle activation in collegiate cross-country runners. Journal of Strength and Conditioning Research, 27(9), 2619-2625. https://doi.org/10.1519/JSC.0b013e31828055d5

Gullett, J. C., Tillman, M. D., Gutierrez, G. M., \& Chow, J. W. (2009). A biomechanical comparison of back and front squats in healthy trained individuals. Journal of Strength and Conditioning Research, 23(1), 284-292. https://doi.org/10.1519/JSC.0b013e31818546bb

Hammond, B., Marques-Bruna, P., Chauhan, E., \& Bridge, C. (2016). Electromyographic activity in superficial muscles of the thigh and hip during the back squat to three different depths with relative loading. Journal of Fitness Research, 5(3), 57-67.

Hutton, B., Salanti, G., Caldwell, D. M., Chaimani, A., Schmid, C. H., Cameron, C., loannidis, J. P. A., Straus, S., Thorlund, K., Jansen, J. P., Mulrow, C., Catalá-López, F., Gøtzsche, P. C., Dickersin, K., Boutron, I., Altman, D. G., \& Moher, D. (2015). The PRISMA Extension Statement for reporting of systematic reviews incorporating Network Meta-analyses of health care interventions: Checklist and explanations. Annals of Internal Medicine, 162(11), 777-784. https://doi.org/10.7326/M14-2385

Iversen, V. M., Mork, P. J., Vasseljen, O., Bergquist, R., \& Fimland, M. S. (2017). Multiple-joint exercises using elastic resistance bands vs. conventional resistance-training equipment: A cross-over study. $\begin{array}{llll}\text { European Journal of } & \text { Sport } \quad \text { Science, } & 17(8), & \end{array}$ https://doi.org/10.1080/17461391.2017.1337229

Kompf, J., \& Arandjelović, O. (2017). The sticking point in the bench press, the squat, and the deadlift: Similarities and differences, and their significance for research and practice. Sports Medicine, 47(4), 631-640. https://doi.org/10.1007/s40279-016-0615-9

Korak, J. A., Paquette, M. R., Fuller, D. K., Caputo, J. L., \& Coons, J. M. (2018). Muscle activation patterns of lower-body musculature among 3 traditional lower-body exercises in trained women. Journal of Strength and Conditioning Research, 32(10), 2770-2775. https://doi.org/10.1519/JSC.0000000000002513

Lynn, S. K., \& Noffal, G. J. (2012). Lower extremity biomechanics during a regular and counterbalanced squat. Journal of Strength and Conditioning Research, 26(9), 2417-2425. https://doi.org/10.1519/JSC.0b013e31823f8c2d

Maher, C. G., Sherrington, C., Herbert, R. D., Moseley, A. M., \& Elkins, M. (2003). Reliability of the PEDro scale for rating quality of randomized controlled trials. Physical Therapy, 83(8), 713-721. https://doi.org/10.1093/ptj/83.8.713

McCaw, S. T., \& Melrose, D. R. (1999). Stance width and bar load effects on leg muscle activity during the parallel squat. Medicine and Science in Sports and Exercise, 31(3), 428-436. https://doi.org/10.1097/00005768-199903000-00012

McCurdy, K. W., Walker, J. L., \& Yuen, D. (2018). Gluteus maximus and hamstring activation during selected weight-bearing resistance exercises. Journal of Strength and Conditioning Research, 32(3), 594-601. https://doi.org/10.1519/JSC.0000000000001893 
Neto, W. K., Soares, E. G., Vieira, T. L., Aguiar, R., Chola, T. A., Sampaio, V. de L., \& Gama, E. F. (2020). Gluteus Maximus Activation during common strength and hypertrophy exercises: A systematic review. Journal of Sports Science \& Medicine, 19(1), 195-203.

Netter, F. H. (1999). Human Anatomy. Elsevier - Health Sciences Division.

Paoli, A., Marcolin, G., \& Petrone, N. (2009). The effect of stance width on the electromyographical activity of eight superficial thigh muscles during back squat with different bar loads. Journal of $\begin{array}{lll}\text { Strength and } \quad \text { Conditioning 246-250. } & \text { Research, }\end{array}$ https://doi.org/10.1519/JSC.0b013e3181876811

Paoli, A., Marcolin, G., \& Petrone, N. (2010). Influence of different ranges of motion on selective recruitment of shoulder muscles in the sitting military press: An electromyographic study. Journal of $\begin{array}{llll}\text { Strength and } \quad \text { Conditioning } & \text { 1578-1583. }\end{array}$ https://doi.org/10.1519/JSC.0b013e3181d756ea

Pereira, G. R., Leporace, G., Chagas, D. das V., Furtado, L. F. L., Praxedes, J., \& Batista, L. A. (2010). Influence of hip external rotation on hip adductor and rectus femoris myoelectric activity during a dynamic parallel squat. Journal of Strength and Conditioning Research, 24(10), 2749-2754. https://doi.org/10.1519/JSC.0b013e3181c6a139

Pham, R. D., Machek, S. B., \& Lorenz, K. A. (2020). Technical aspects and applications of the low-bar back squat. Strength \& Conditioning Journal, 42(3), 121-128. https://doi.org/10.1519/SSC.0000000000000521

Robbins, D. (2011). A comparison of muscular activation during the back squat and deadlift to the countermovement jump [Master Thesis, Sacred Heart University].

Schaefer, L. V., \& Bittmann, F. N. (2017). Are there two forms of isometric muscle action? Results of the experimental study support a distinction between a holding and a pushing isometric muscle function. BMC Sports Science, Medicine and Rehabilitation, 9(1), 11. https://doi.org/10.1186/s13102-017$\underline{0075-z}$

Schoenfeld, B. J. (2010). Squatting kinematics and kinetics and their application to exercise performance. Journal of Strength and Conditioning Research, 24(12), 3497-3506. https://doi.org/10.1519/JSC.0b013e3181bac2d7

Schwanbeck, S., Chilibeck, P. D., \& Binsted, G. (2009). A comparison of free weight squat to Smith machine squat using electromyography. Journal of Strength and Conditioning Research, 23(9), 2588-2591. https://doi.org/10.1519/JSC.0b013e3181b1b181

Sinclair, J., Taylor, P. J., Hebron, J., Brooks, D., Hurst, H. T., \& Atkins, S. (2015). The reliability of electromyographic normalization methods for cycling analyses. Journal of Human Kinetics, 46(1), 19-27. https://doi.org/10.1515/hukin-2015-0030

Urrútia, G., \& Bonfill, X. (2010). Declaración PRISMA: Una propuesta para mejorar la publicación de revisiones sistemáticas y metaanálisis. Medicina Clínica, 135(11), 507-511. https://doi.org/10.1016/j.medcli.2010.01.015

Van den Tillaar, R., Andersen, V., \& Saeterbakken, A. H. (2014). Comparison of muscle activation and performance during 6 RM two legged free weight squats. Kinesiologia Slovenica, 20(2), 5-16.

Vigotsky, A. D., Bryanton, M. A., Nuckols, G., Beardsley, C., Contreras, B., Evans, J., \& Schoenfeld, B. J. (2019). Biomechanical, anthropometric, and psychological determinants of barbell back squat strength. Journal of Strength and Conditioning Research, 33(7S), S26-S35. https://doi.org/10.1519/JSC.0000000000002535

Wretenberg, P., Feng, Y., \& Arborelius, U. P. (1996). High- and low-bar squatting techniques during weight-training. Medicine and Science in Sports and Exercise, 28(2), 218-224. https://doi.org/10.1097/00005768-199602000-00010 
Wright, G. A., Delong, T. H., \& Gehlsen, G. (1999). Electromyographic activity of the hamstrings during performance of the leg curl, stiff-leg deadlift, and back squat movements. Journal of Strength and Conditioning Research, 13(2), 168-174. https://doi.org/10.1519/00124278-199905000-00012

Wu, H.-W., Tsai, C.-F., Liang, K.-H., \& Chang, Y.-W. (2019). Effect of loading devices on muscle activation in squat and lunge. Journal of Sport Rehabilitation, 29(2), 200-205. https://doi.org/10.1123/jsr.2018-0182

Yavuz, H. U., Erdağ, D., Amca, A. M., \& Aritan, S. (2015). Kinematic and EMG activities during front and back squat variations in maximum loads. Journal of Sports Sciences, 33(10), 1058-1066. https://doi.org/10.1080/02640414.2014.984240 